\title{
Base-free Dynamic Kinetic Resolution of Secondary Alcohols with a Ruthenium-Lipase Couple
}

\author{
Inyeol Yun, Jin Yong Park, Jaiwook Park*, and Mahn-Joo Kim*
}

Department of Chemistry, Pohang University of Science and Technology 77 Cheongam-ro, Pohang 37673, Republic of Korea

E-mail:mjkim@postech.ac.kr

\section{Supporting Information}

\author{
Table of Contents
}

1. ${ }^{1} \mathrm{H}$ and ${ }^{13} \mathrm{C}$ NMR spectra of DKR products

S2-S18

2. HPLC chromatograms of DKR products

S19-S35 
1. ${ }^{1} \mathrm{H}$ and ${ }^{13} \mathrm{C}$ NMR spectra of DKR products

\section{(R)-1-Phenylethyl acetate (13a):}

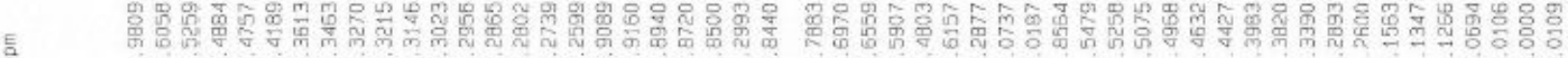

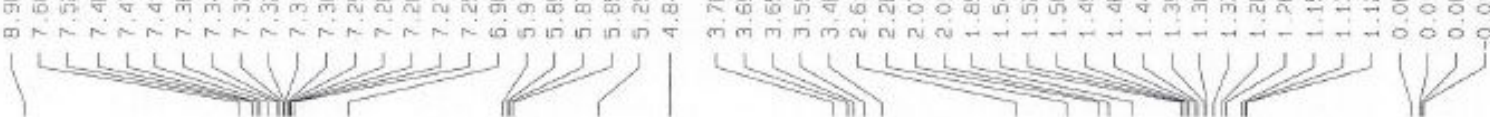

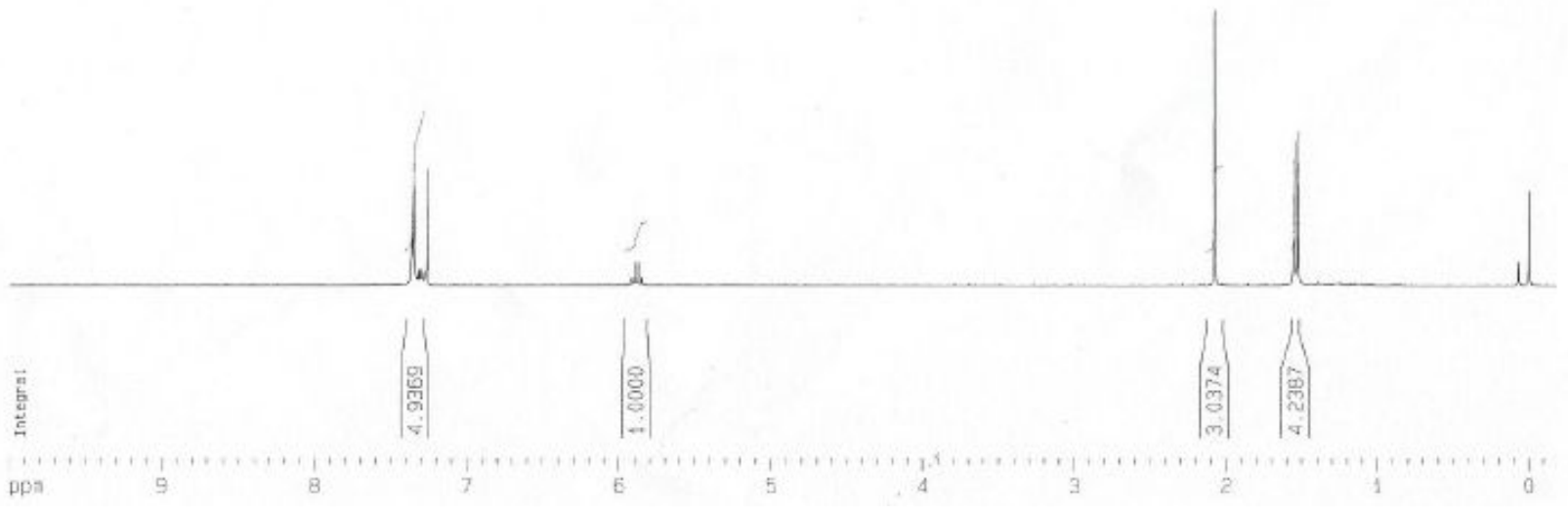

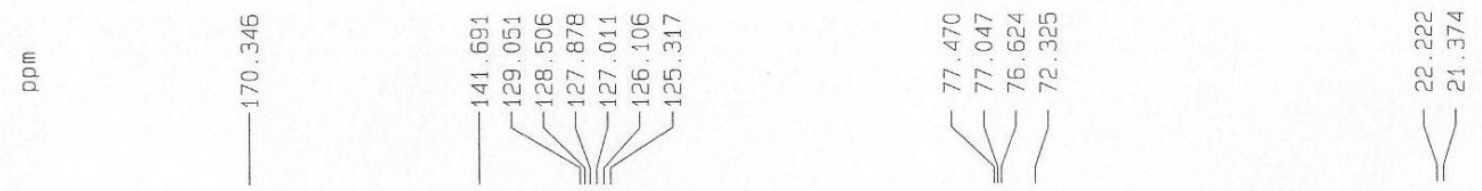

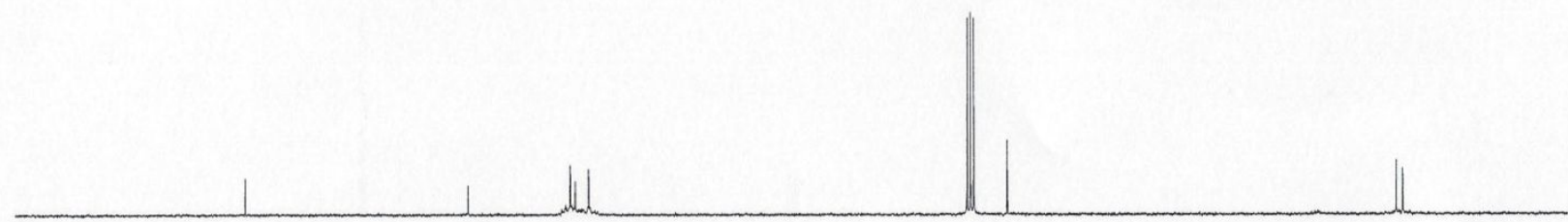




\section{(R)-1-(p-Tolyl)ethyl acetate (13b):}
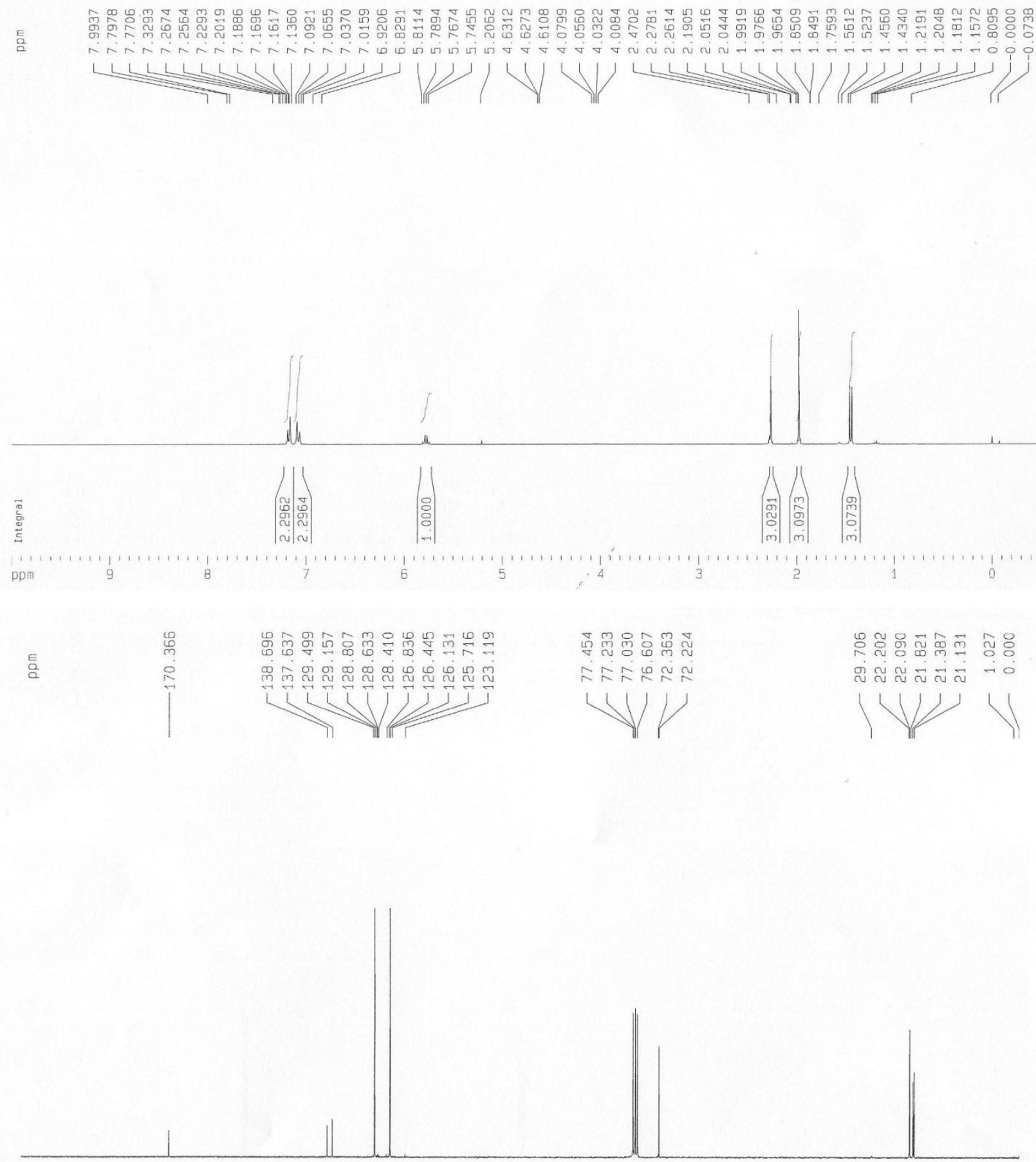
(R)-1-(4-Methoxyphenyl)ethyl acetate (13c):

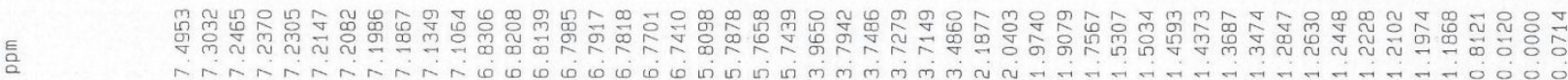

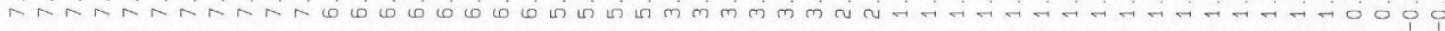

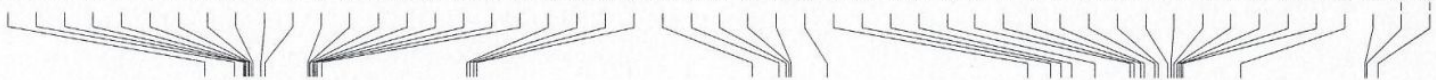
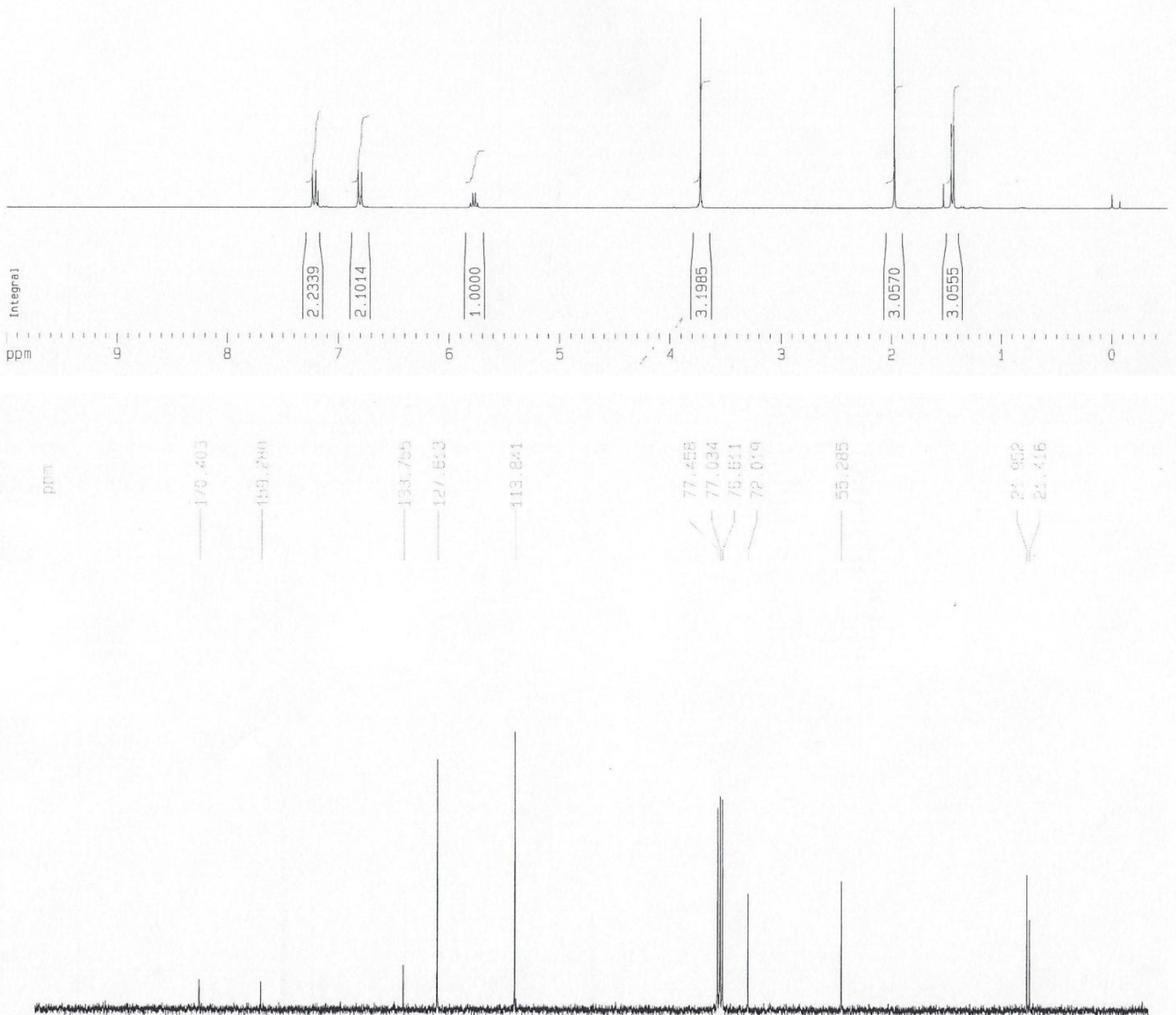
(R)-1-(4-Fluorophenyl)ethyl acetate (13d):

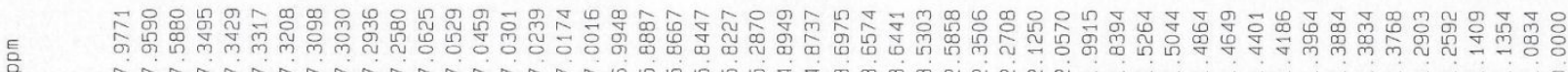

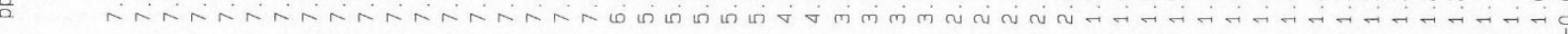
$L^{L L L L L L U}$

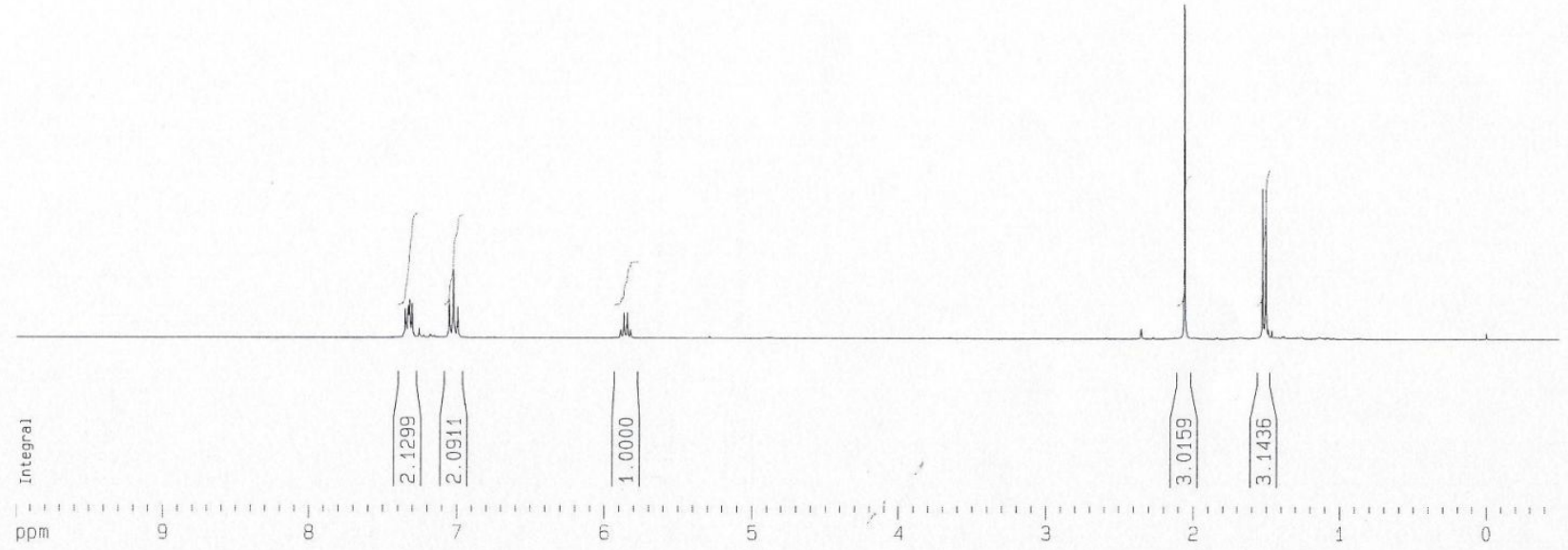

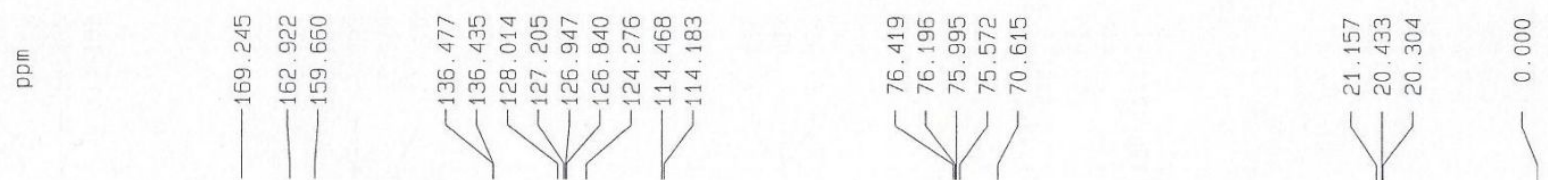

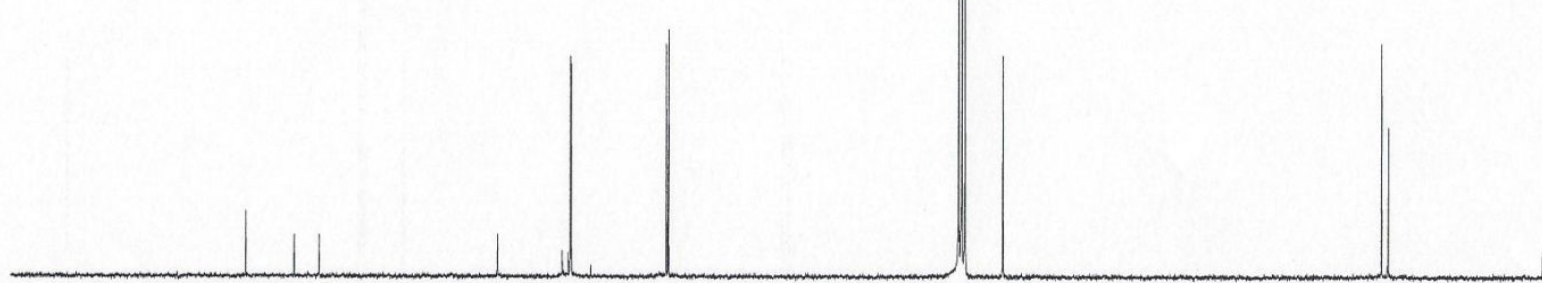


(R)-1-(4-Chlorophenyl)ethyl acetate (13e):

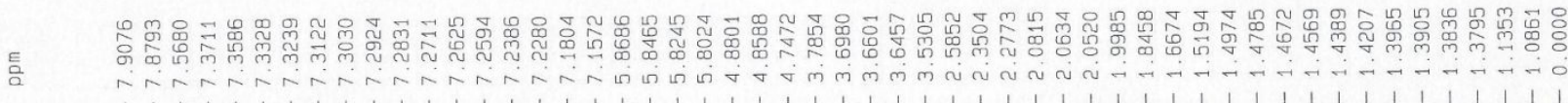

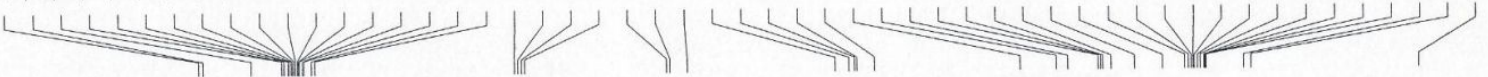
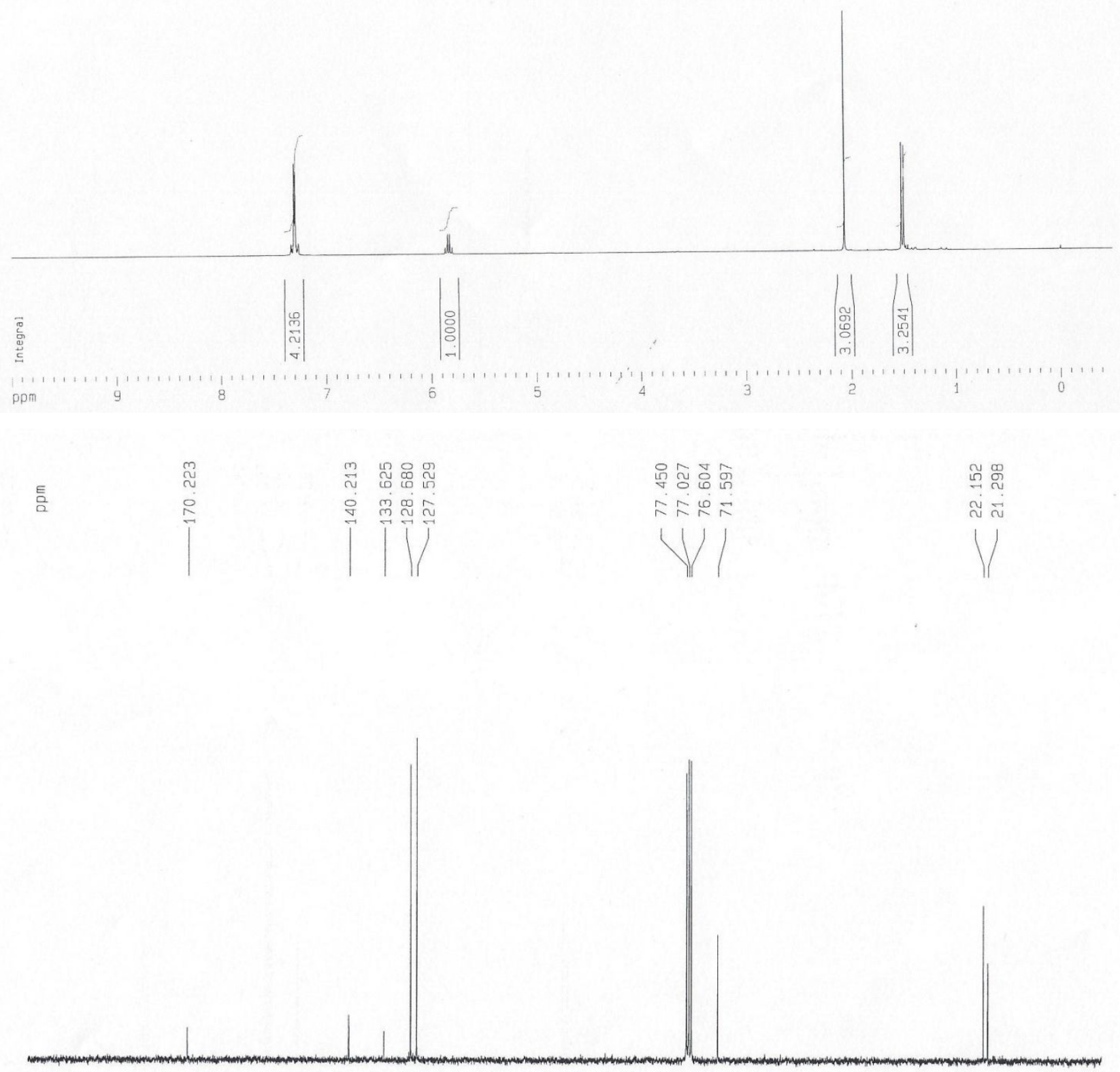
(R)-1-(4-Bromophenyl)ethyl acetate (13f):
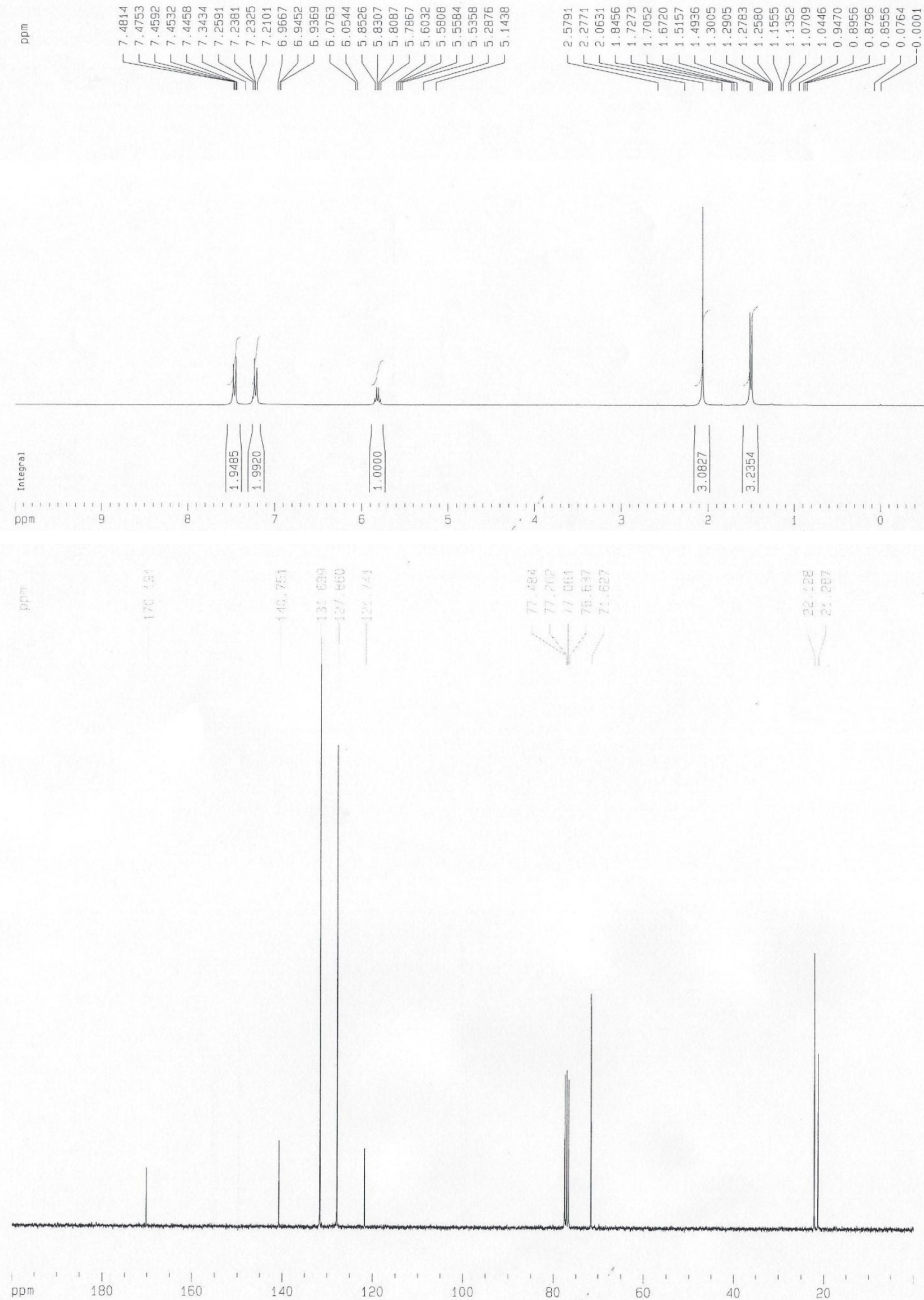
(R)-1-(3-Chlorophenyl)ethyl acetate (13g):
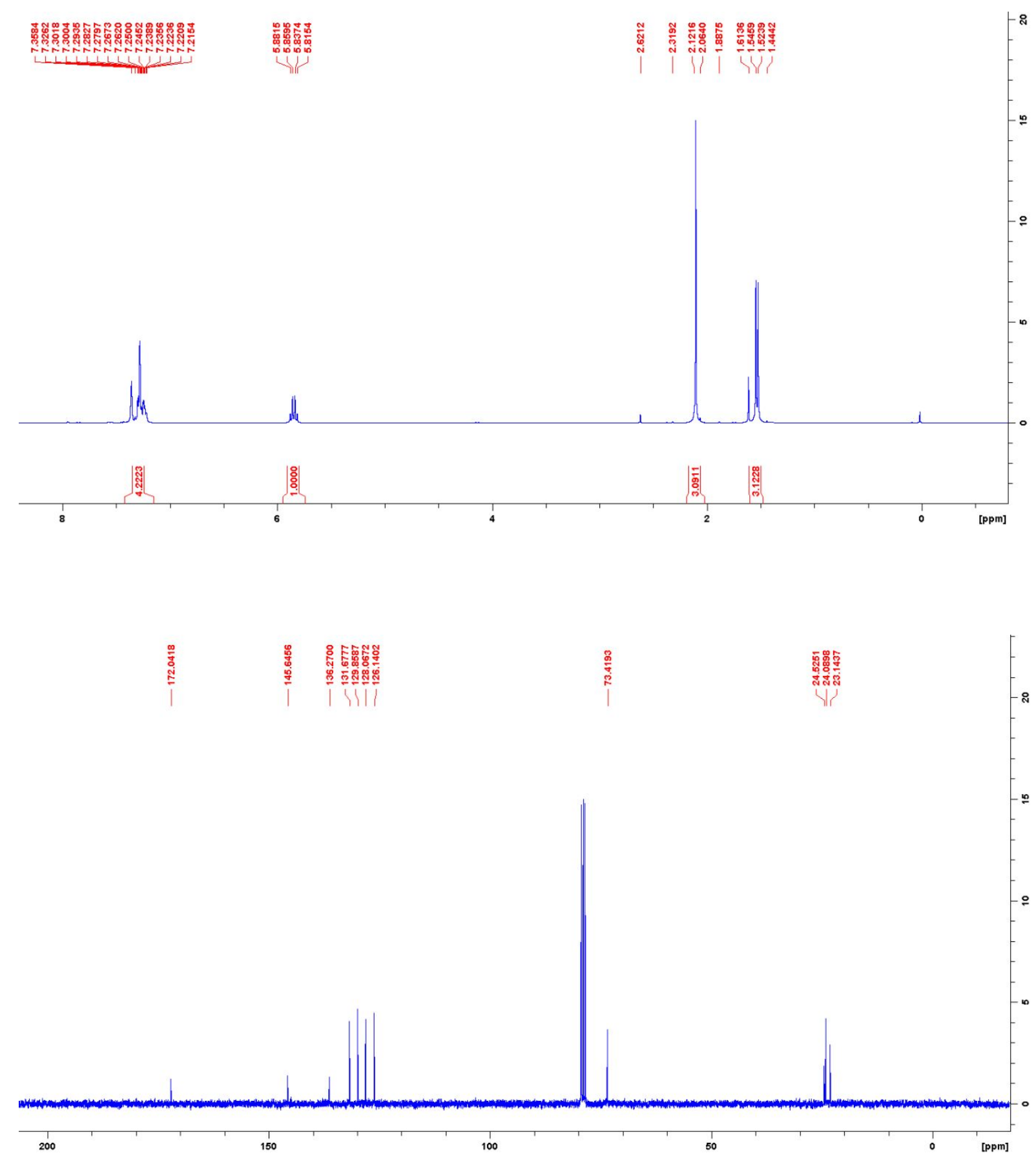


\section{(R)-3-Chloro-1-phenylpropyl acetate (13h):}

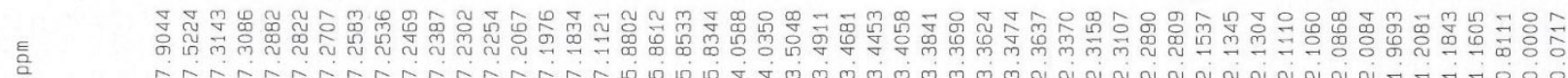

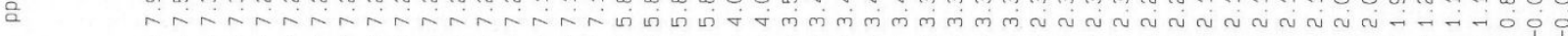
L

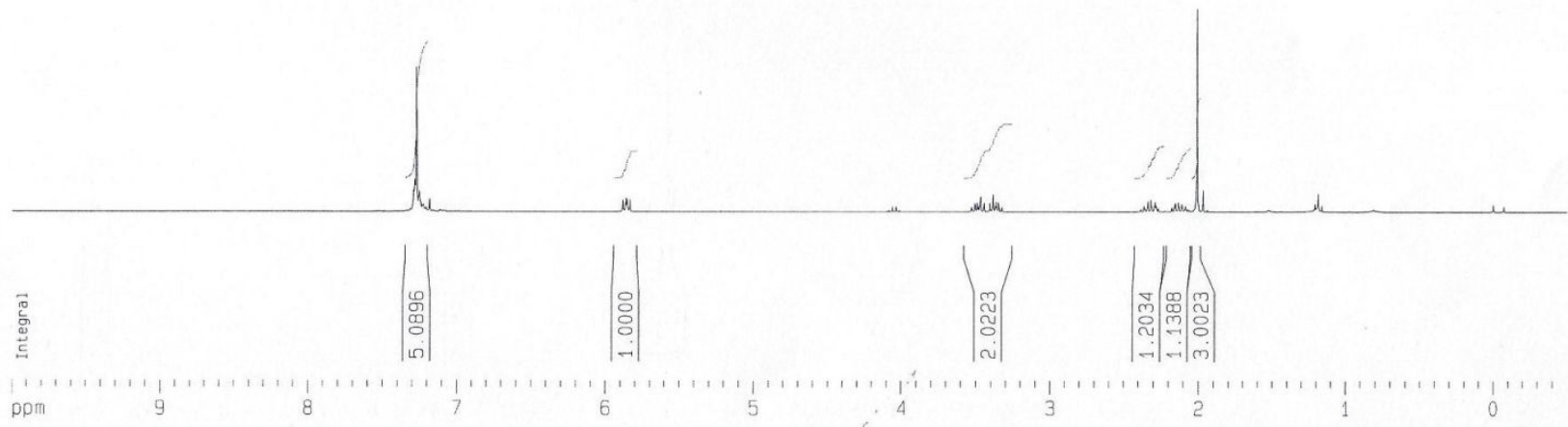

言

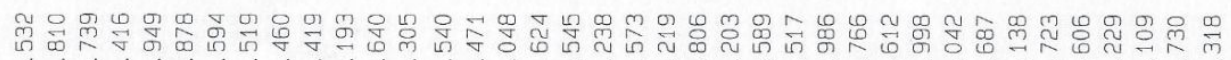
gु

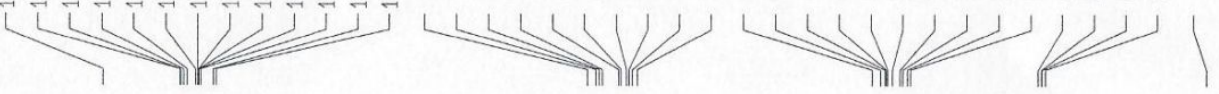

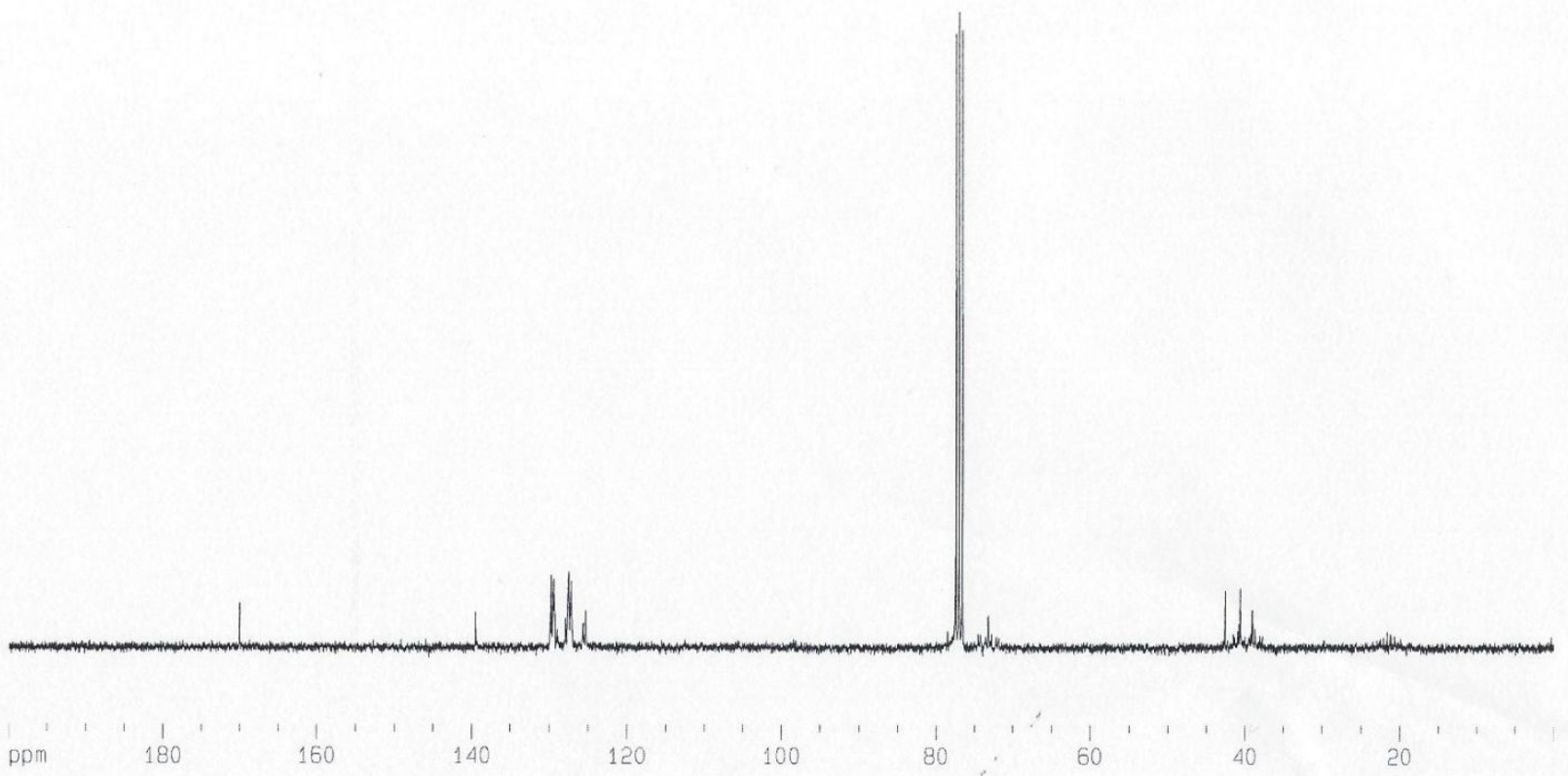


(R)-1-Phenylpropan-2-yl acetate (13i):

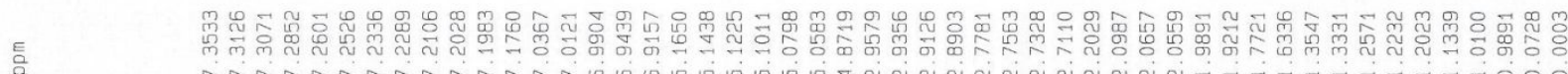
ShA

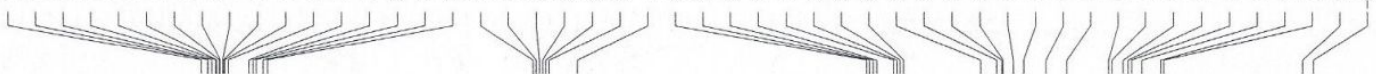
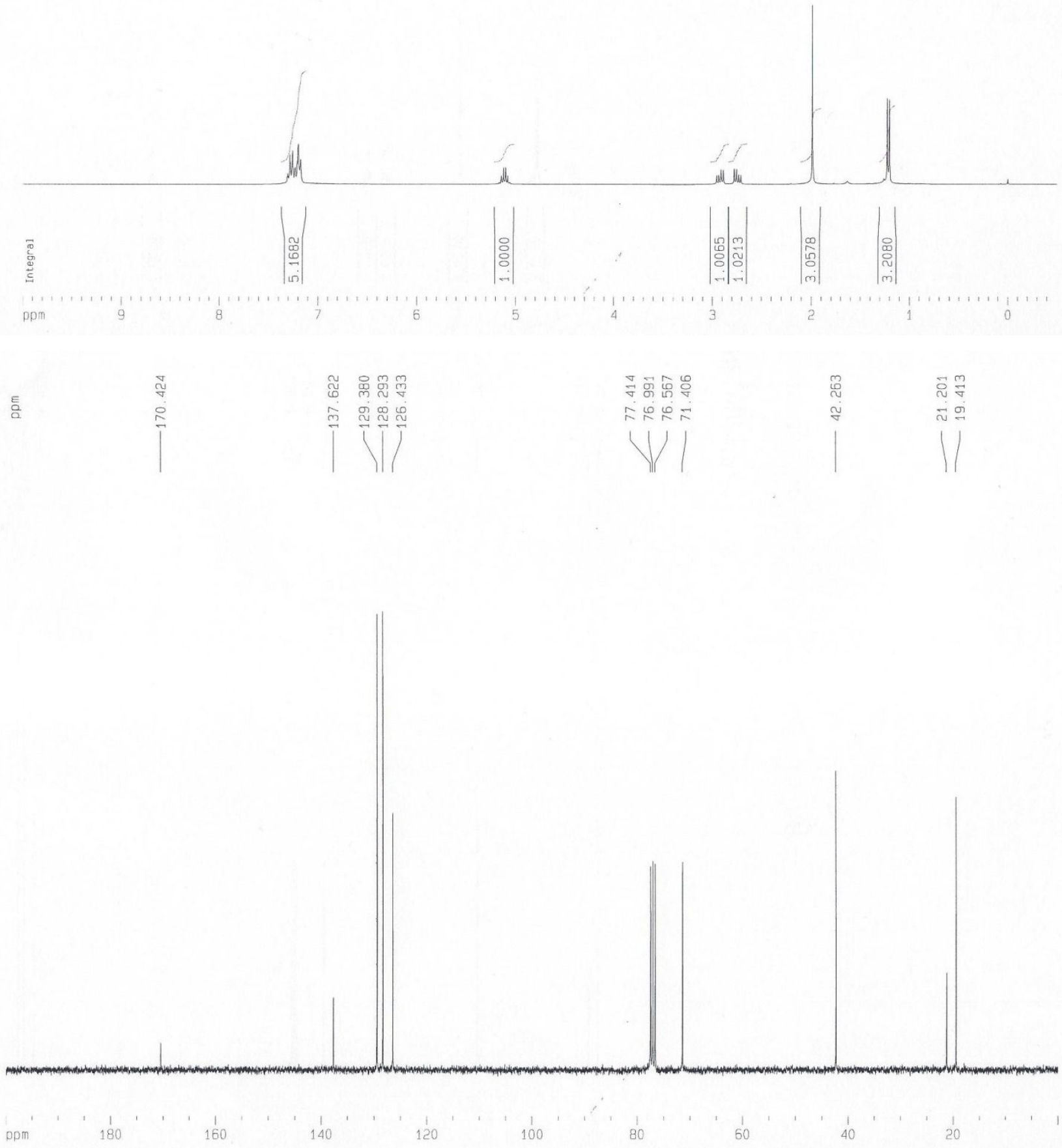
(R)-4-Phenylbutan-2-yl acetate (13j):

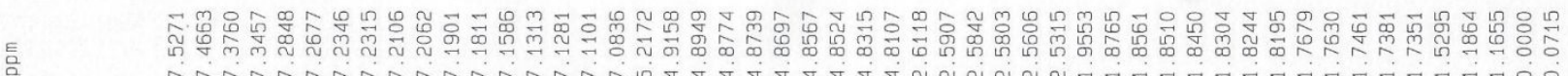

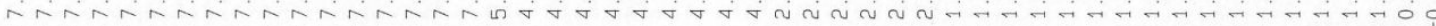
LLLU

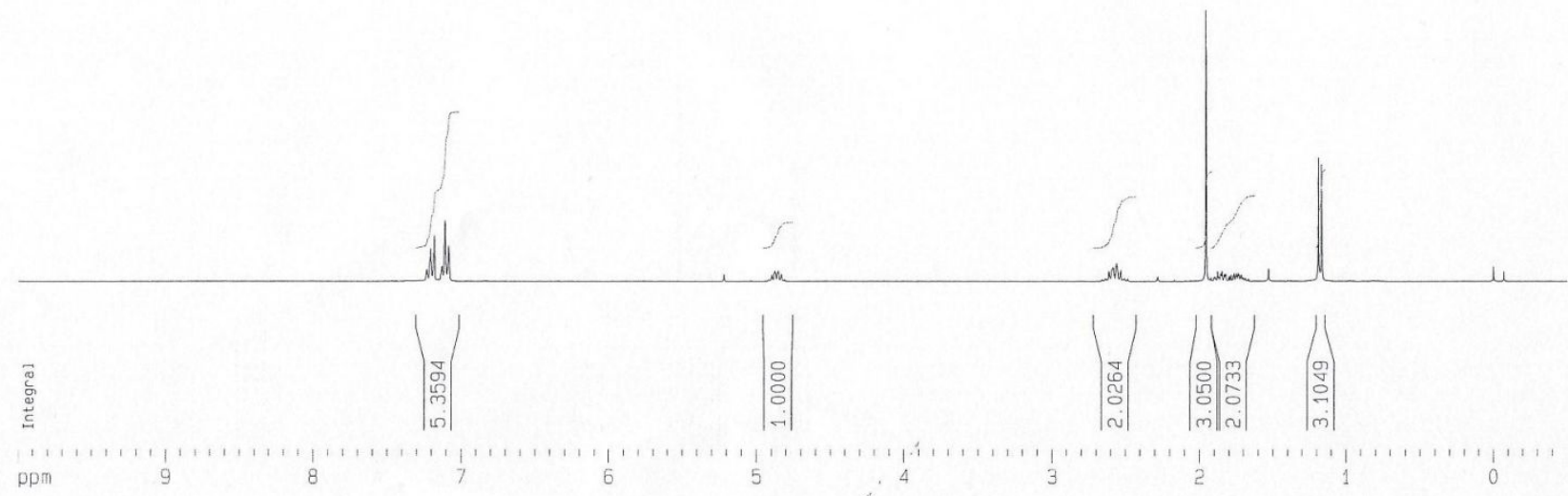

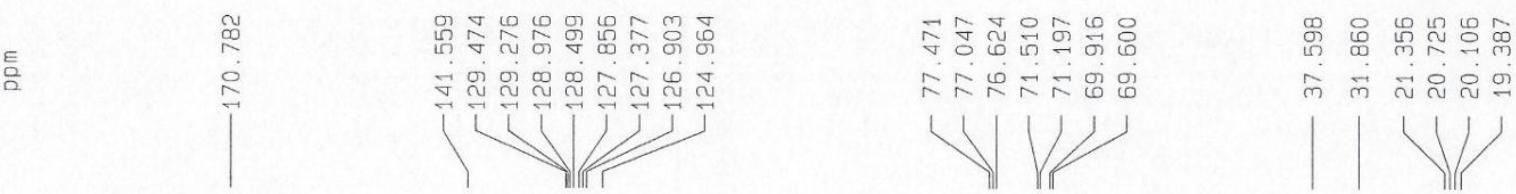

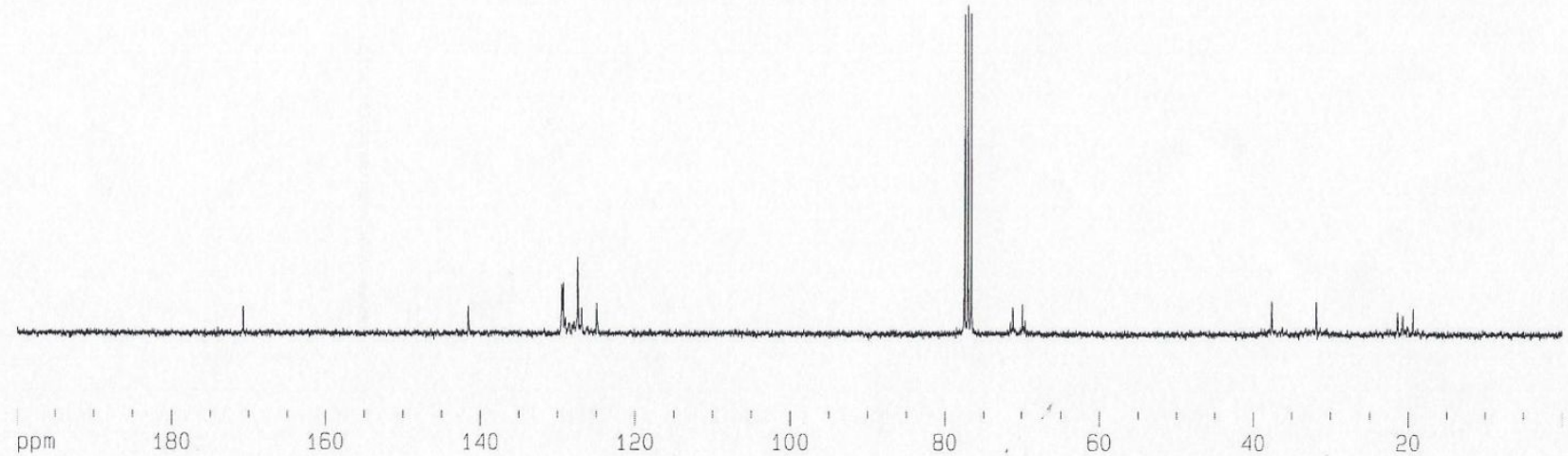




\section{(R)-1-(Naphthalen-2-yl)ethyl acetate (13k):}

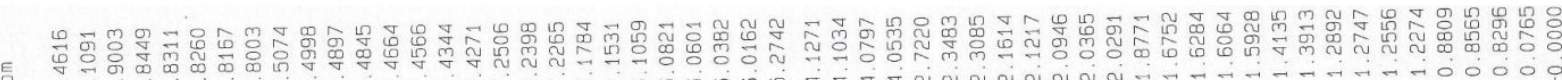
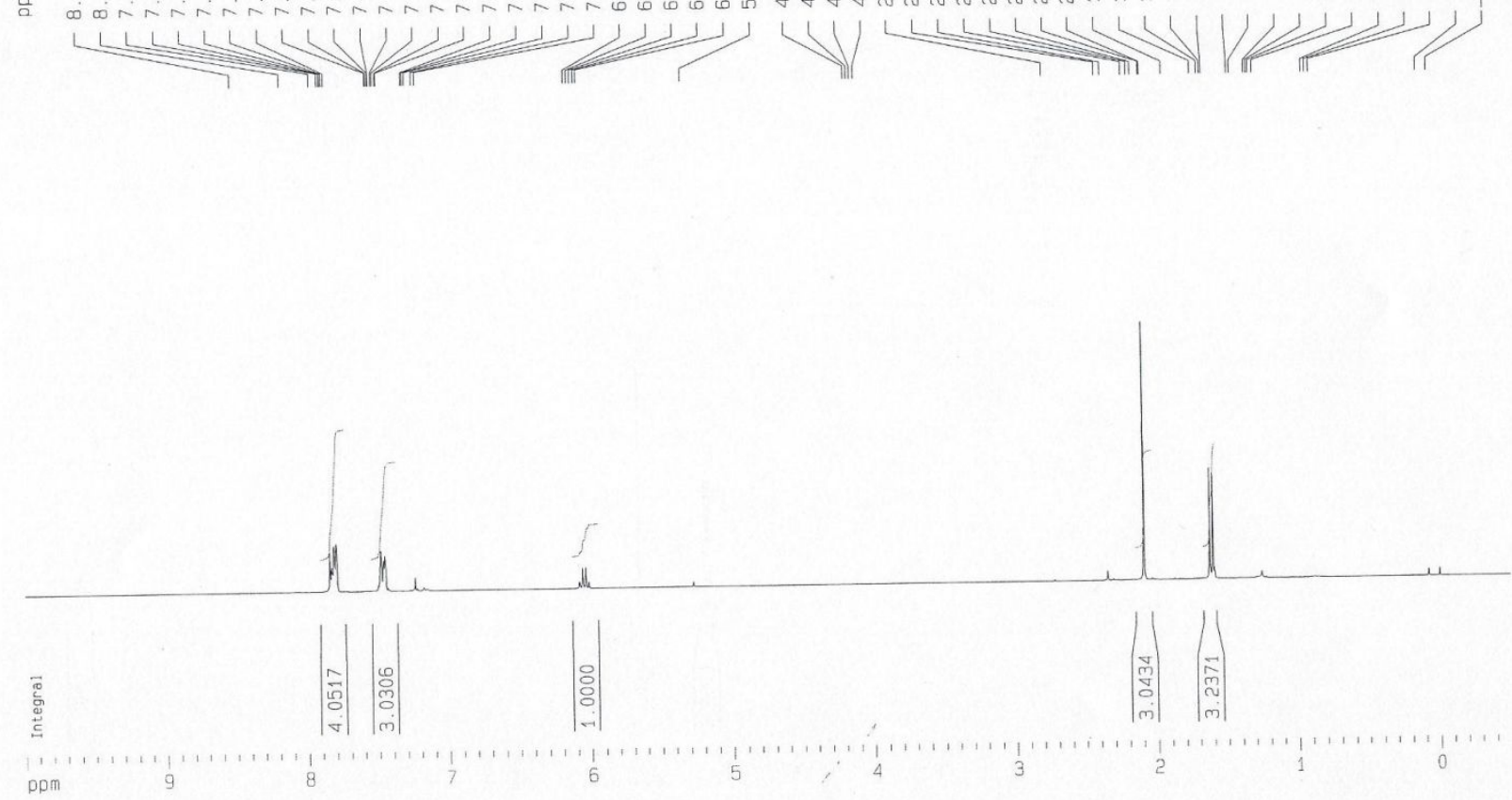

高

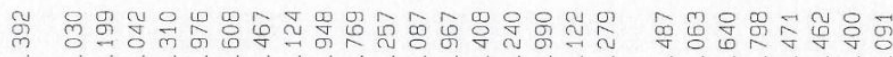

L

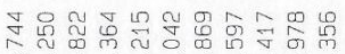

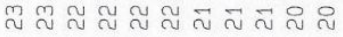
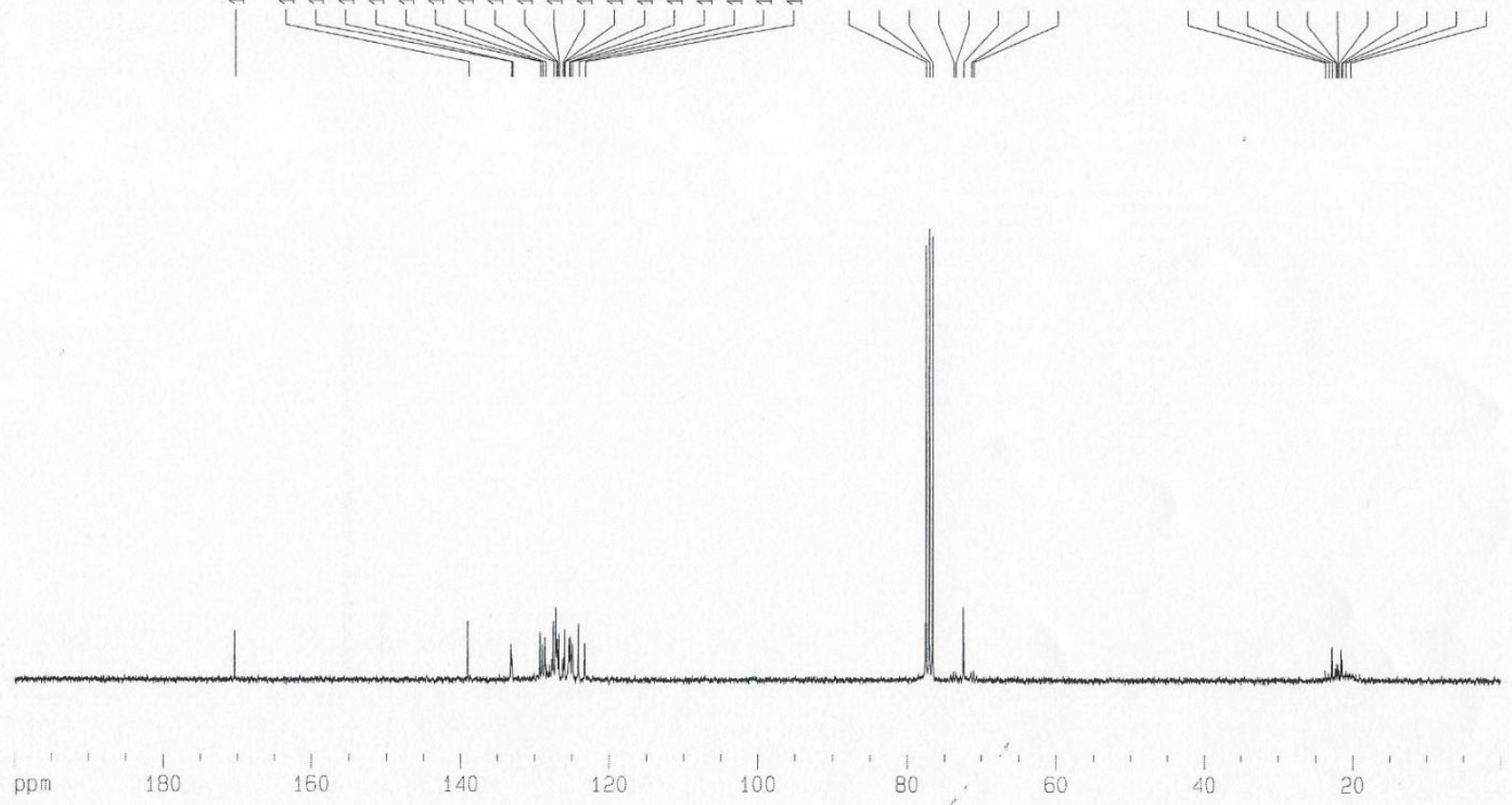
(R)-1-Phenylallyl acetate (13l):

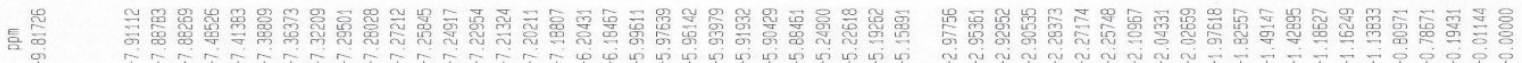

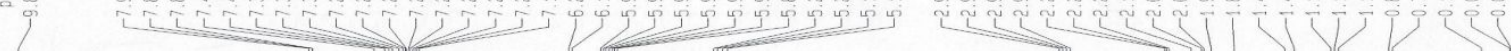

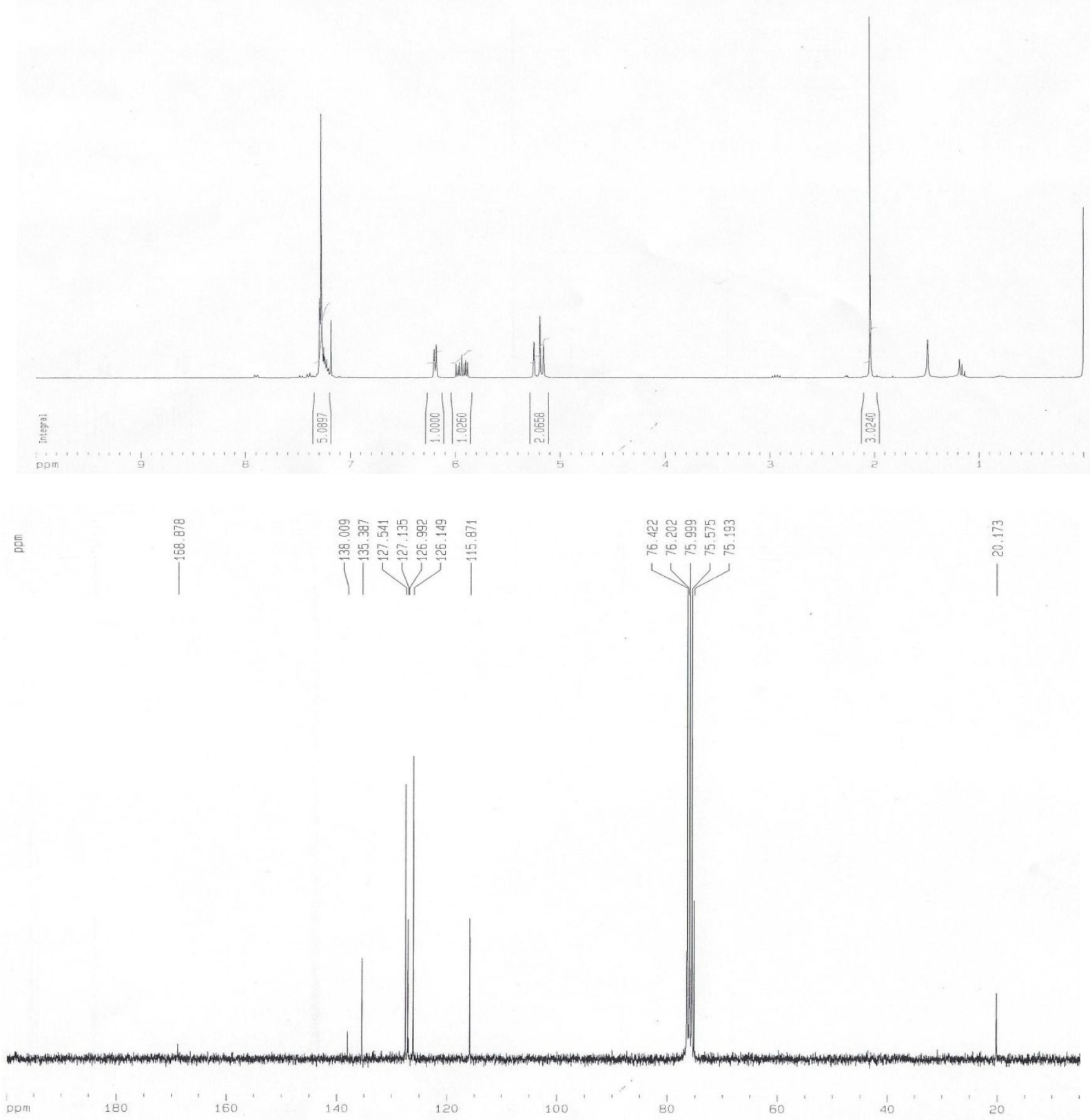


(R)-1-(p-Tolyl)allyl acetate (13m):

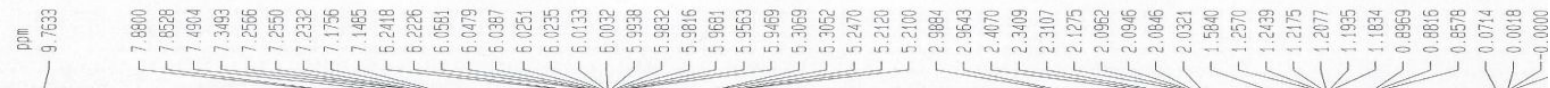

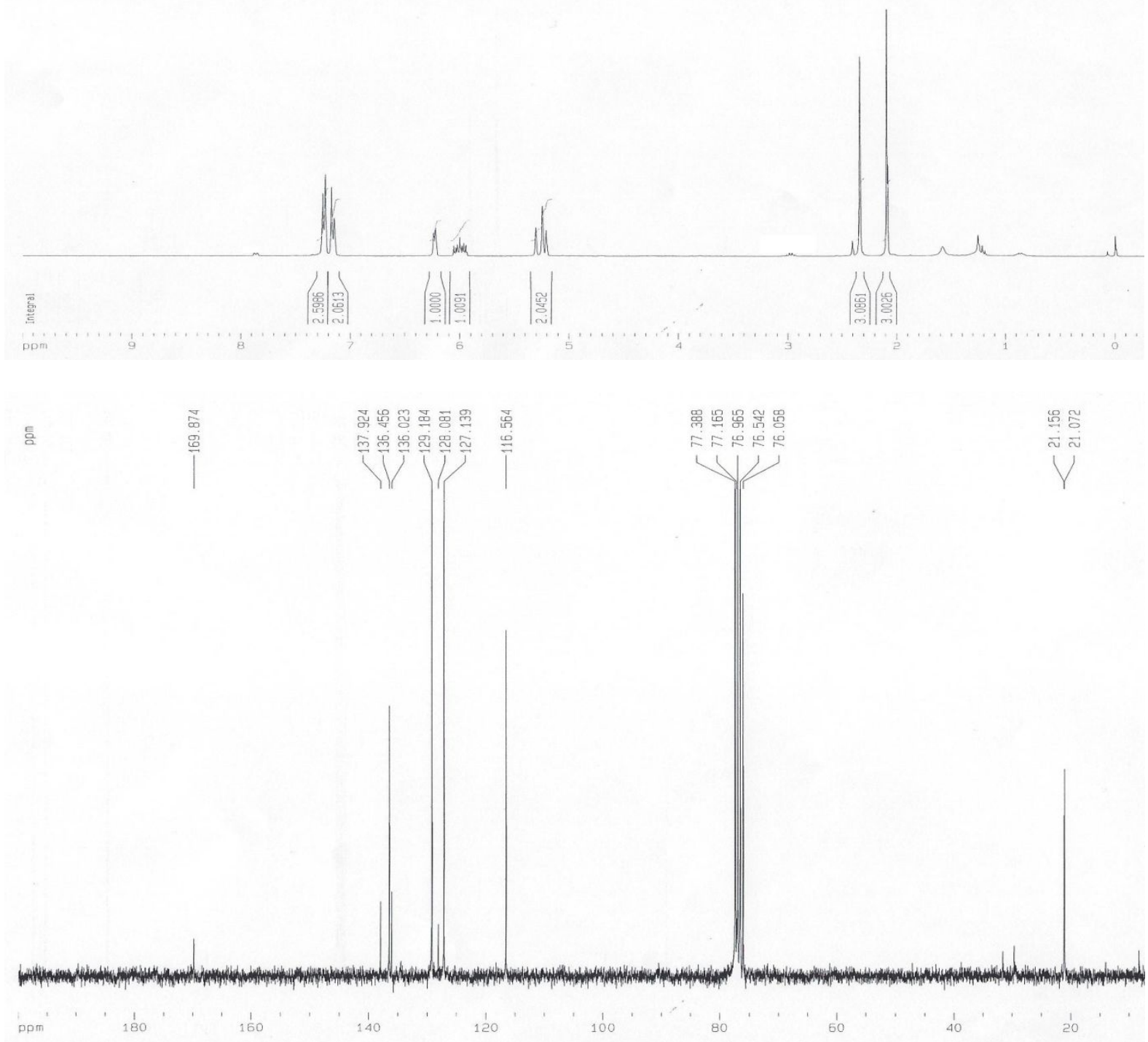


(R)-1-(4-Methoxyphenyl)allyl acetate (13n):

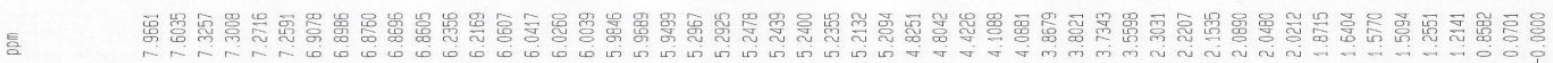

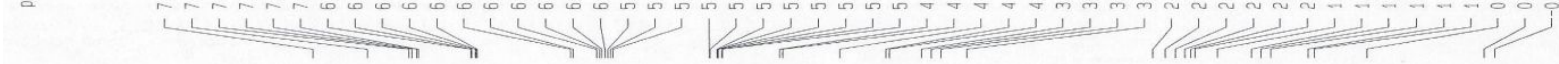

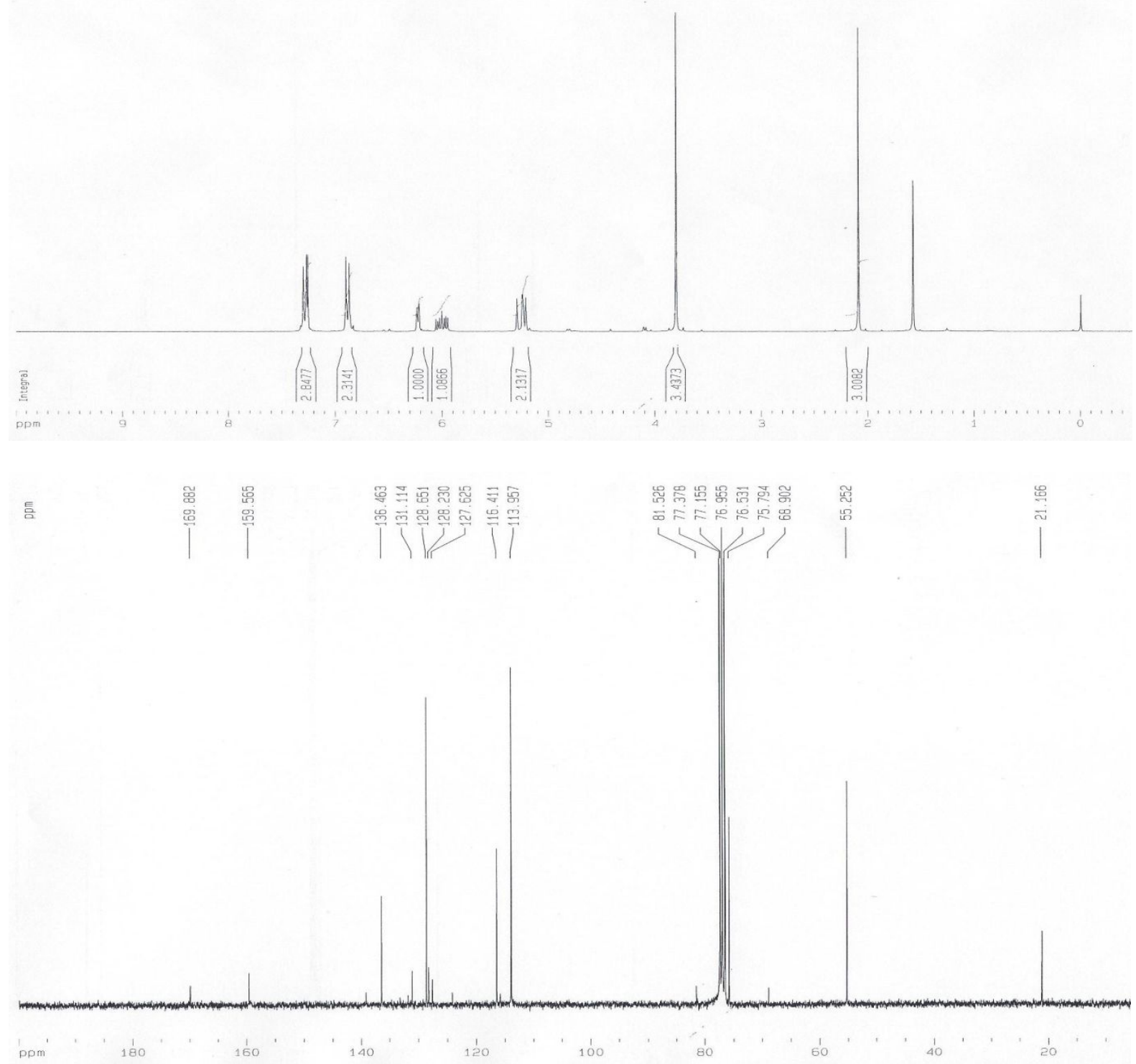


(R)-1-(4-Chlorophenyl)allyl acetate (13o):

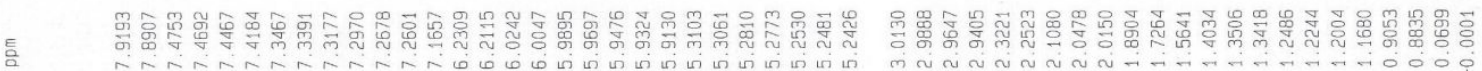
(1)
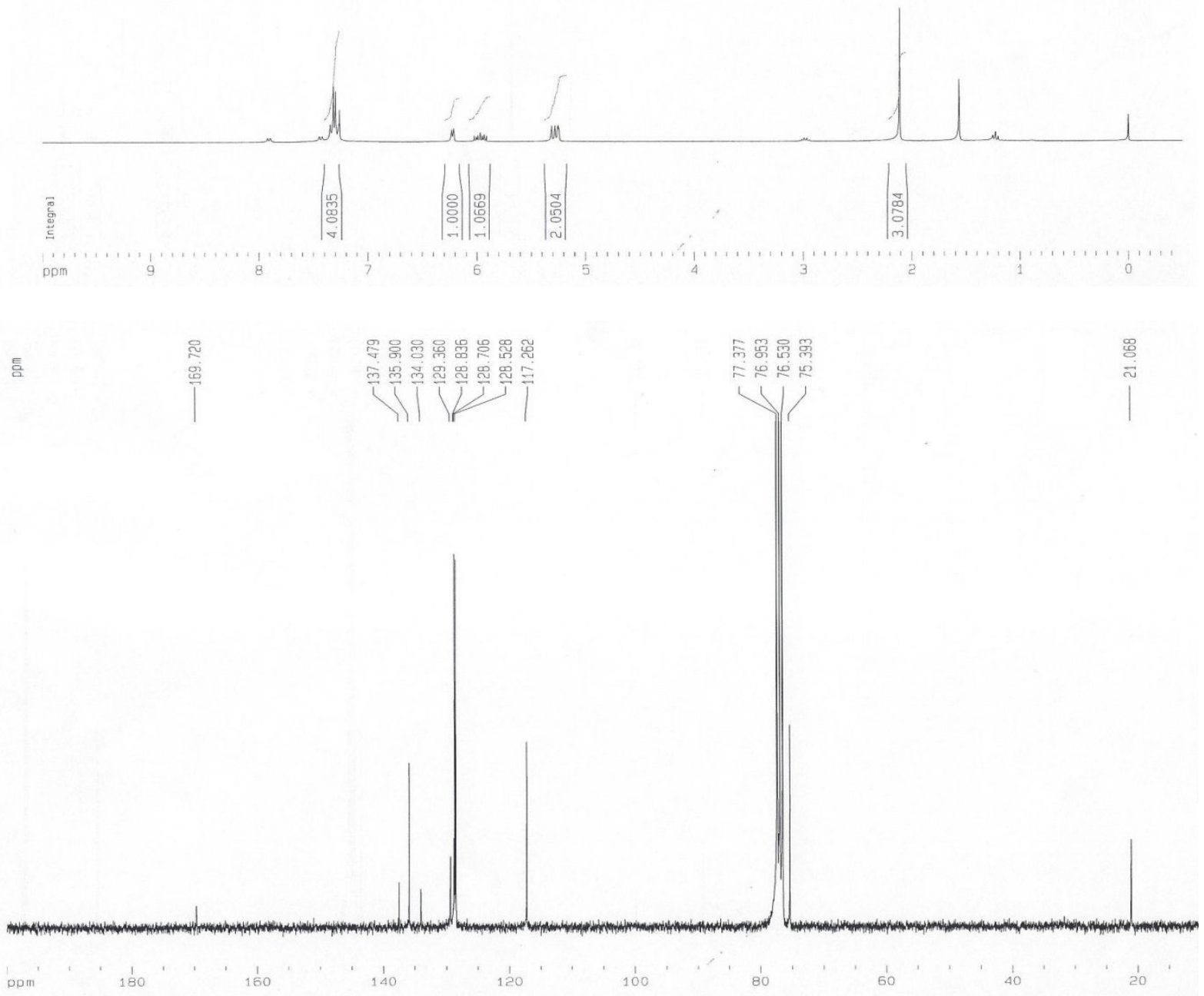
(R)-1-(4-Bromophenyl)allyl acetate (13p):

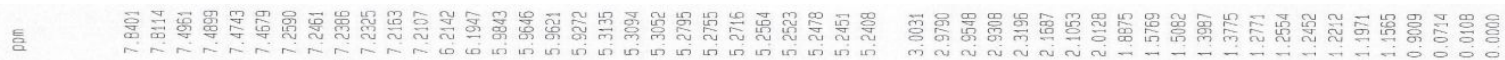
20
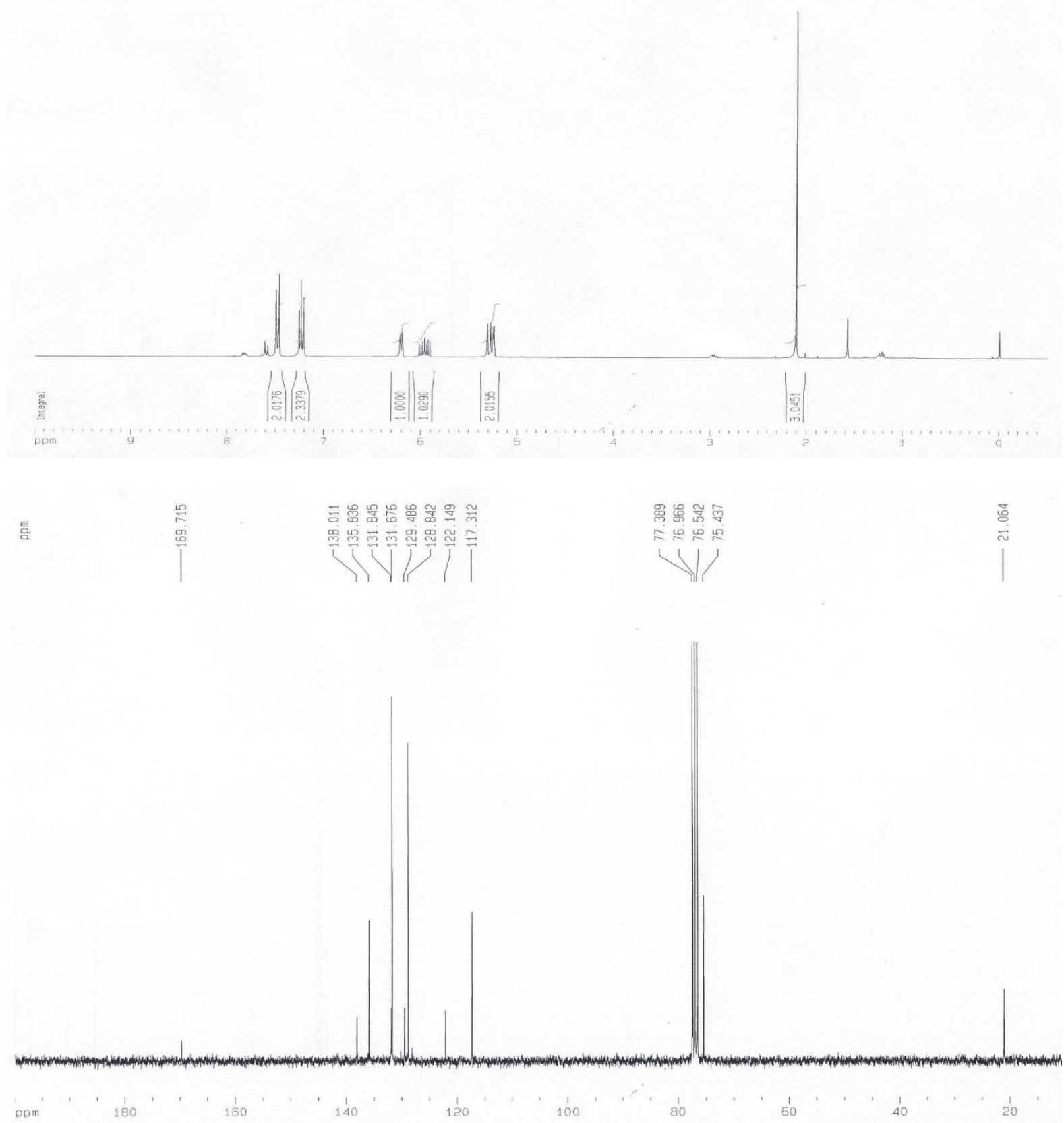
$(R, E)-4-P h e n y l b u t-3-e n-2-y l$ acetate (13q):

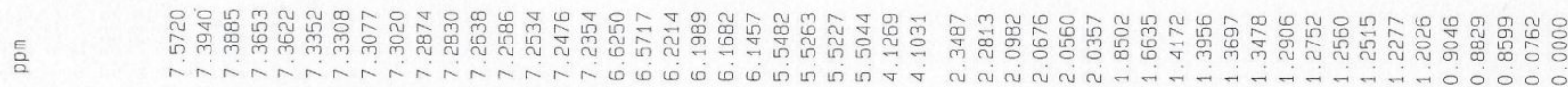
(I)
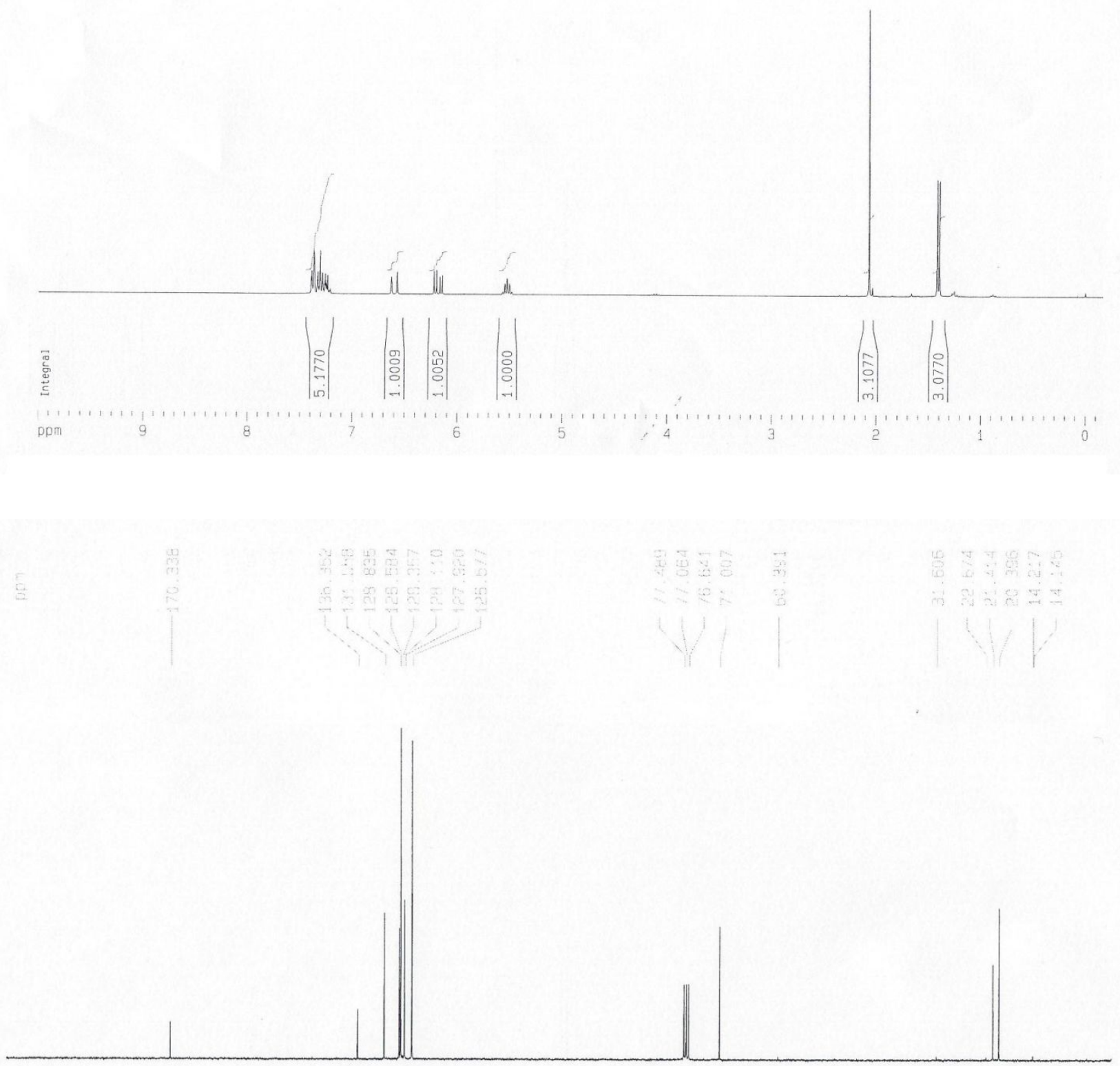


\section{HPLC chromatograms of DKR products}

\section{$(R / S)$ - and $(R)-1-P h e n y l e t h y l$ acetate (13a):}

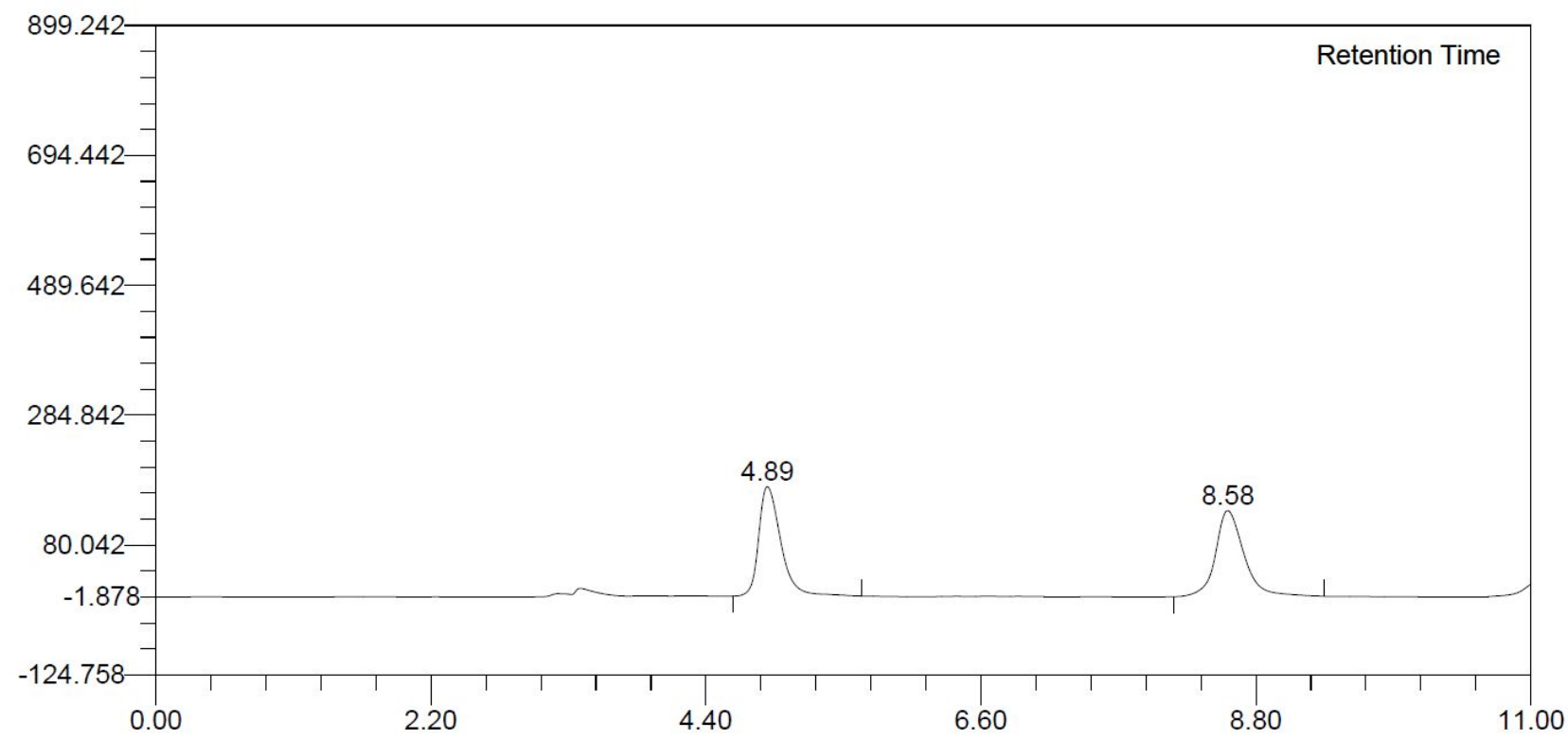

\begin{tabular}{|c|r|r|r|r|r|r|r|}
\hline PK No. & RT & Peak Width & Area & Height & Area \% & Height \% & Code \\
\hline 1 & 4.893 & 0.182 & 42562930 & 172654 & 49.72137 & 56.01612 & BB \\
2 & 8.576 & 0.227 & 43039955 & 135568 & 50.27863 & 43.98388 & BB \\
\hline
\end{tabular}

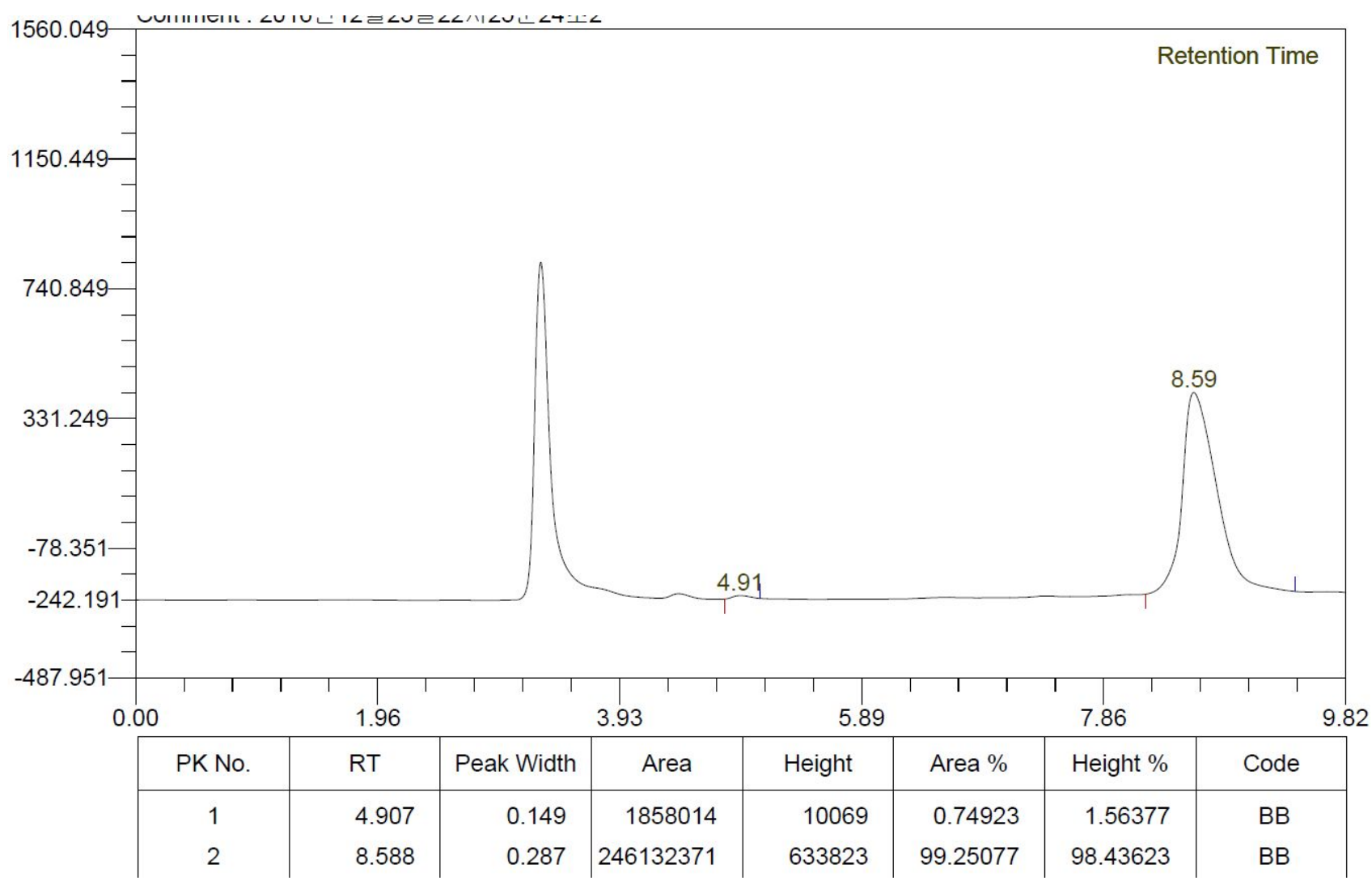


$(R / S)$ - and $(R)-1-(p-T o l y l) e t h y l$ acetate (13b):

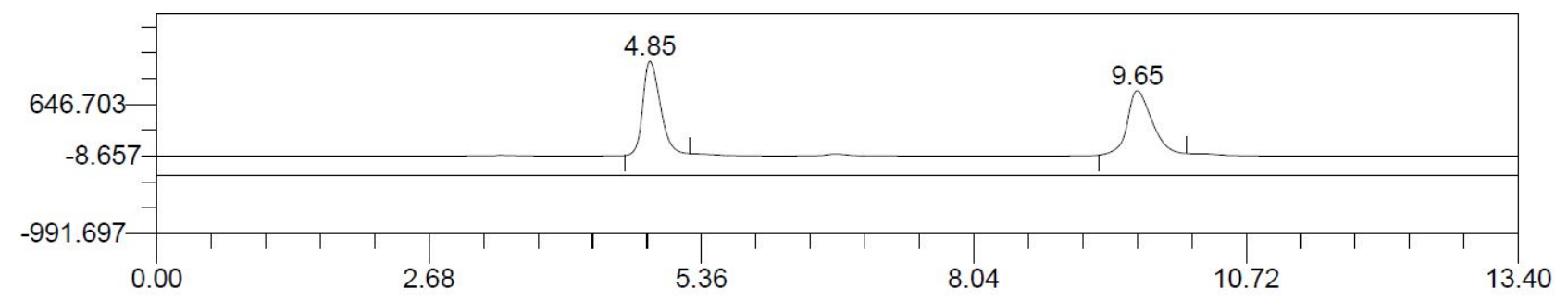

\begin{tabular}{|c|c|r|c|r|r|r|r|}
\hline PK No. & RT & Peak Width & Area & Height & Area \% & Height \% & Code \\
\hline 1 & 4.853 & 0.181 & 280866252 & 1185651 & 51.18668 & 59.49513 & BB \\
2 & 9.648 & 0.247 & 267843381 & 807203 & 48.81332 & 40.50487 & BB
\end{tabular}

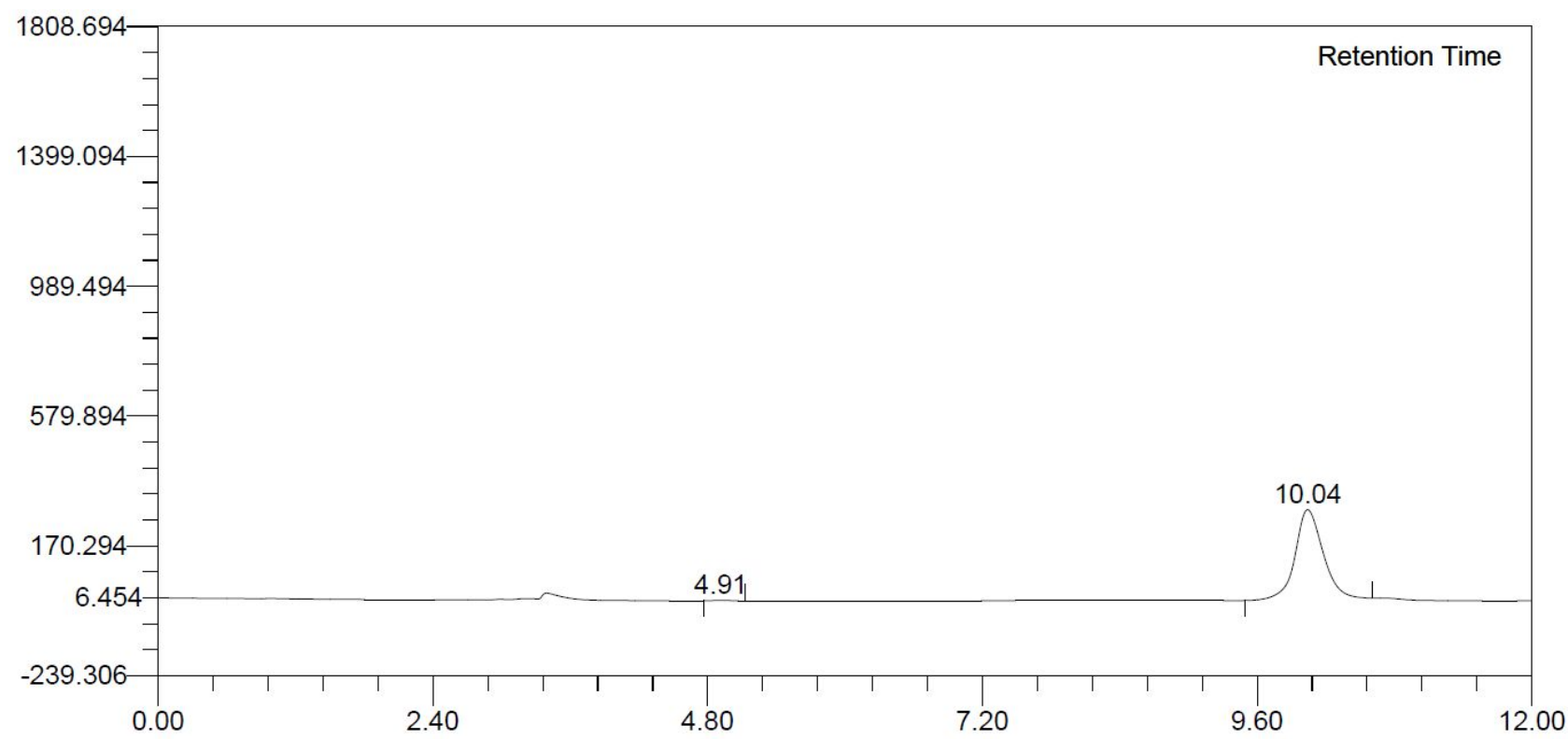

\begin{tabular}{|c|r|r|r|r|r|r|r|}
\hline PK No. & \multicolumn{1}{|c|}{ RT } & Peak Width & \multicolumn{1}{c|}{ Area } & Height & \multicolumn{1}{|c|}{ Area \% } & Height \% & Code \\
\hline 1 & 4.913 & 0.162 & 276853 & 1387 & 0.28829 & 0.48790 \\
2 & 10.043 & 0.247 & 95755684 & 282892 & 99.71171 & 99.51210 & BB
\end{tabular}


$(R / S)$ - and (R)-1-(4-Methoxyphenyl)ethyl acetate (13c):

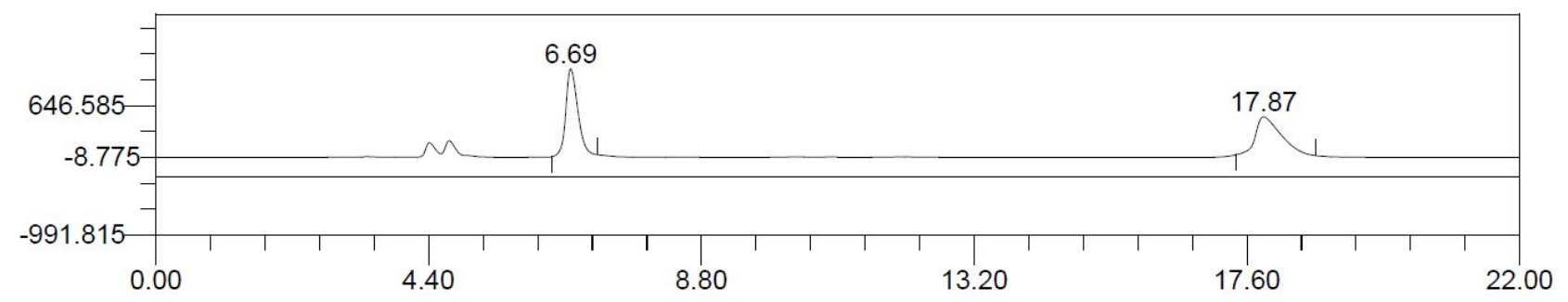

\begin{tabular}{|c|r|r|c|r|r|r|r|}
\hline PK No. & \multicolumn{1}{|c|}{ RT } & Peak Width & Area & Height & Area \% & Height \% & Code \\
\hline 1 & 6.693 & 0.200 & 294923296 & 1104126 & 51.46575 & 69.52392 & BB \\
2 & 17.872 & 0.430 & 278124441 & 483998 & 48.53425 & 30.47608 & BB
\end{tabular}

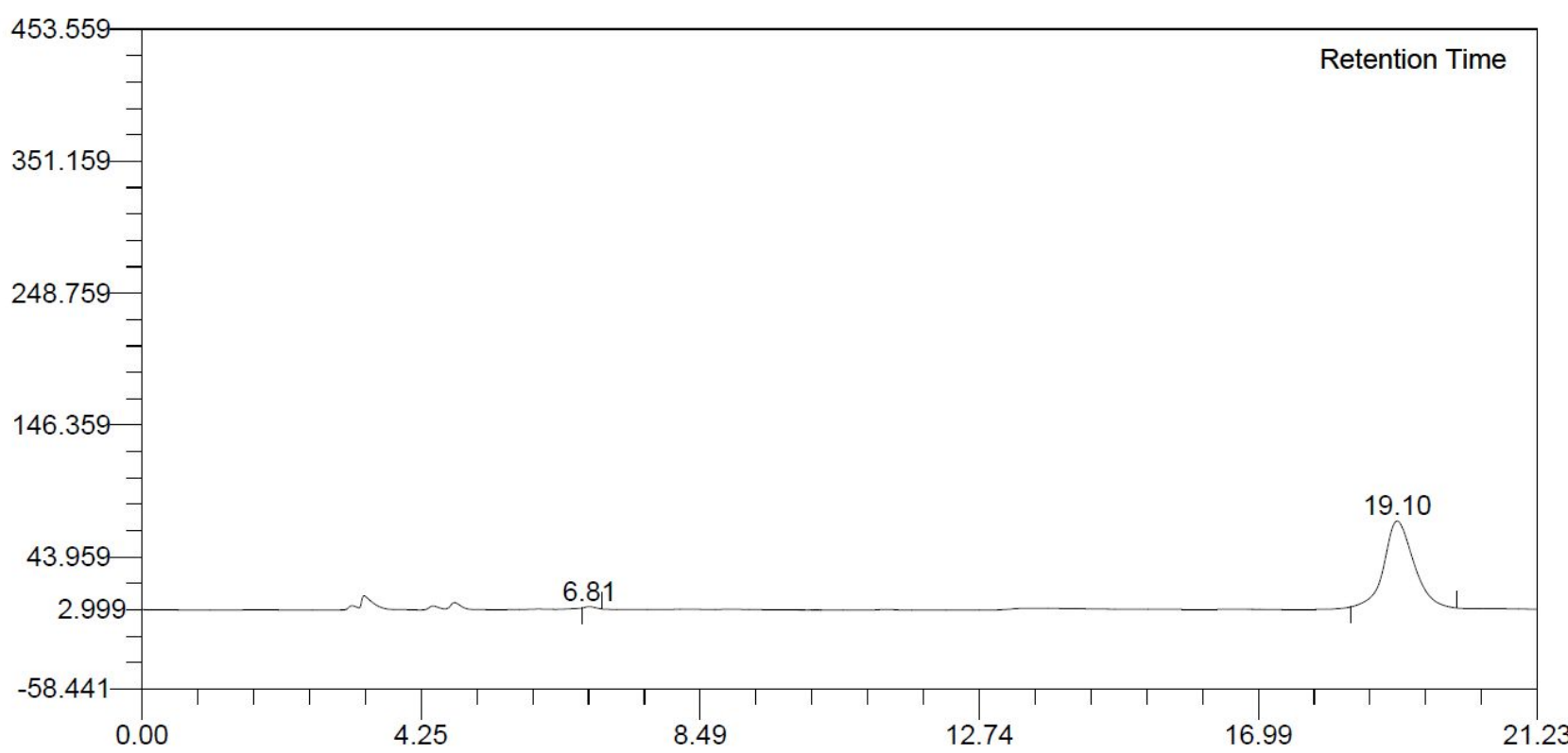

\begin{tabular}{|c|r|r|r|r|r|r|r|}
\hline PK No. & \multicolumn{1}{|c|}{ RT } & Peak Width & \multicolumn{1}{c|}{ Area } & Height & \multicolumn{1}{|c|}{ Area \% } & Height \% & Code \\
\hline 1 & 6.807 & 0.134 & 274451 & 1413 & 0.65192 & 2.06440 \\
2 & 19.099 & 0.458 & 41824209 & 67033 & 99.34808 & 97.93560 & BB \\
\hline
\end{tabular}




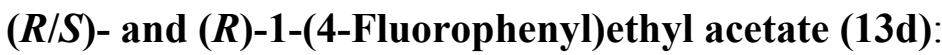

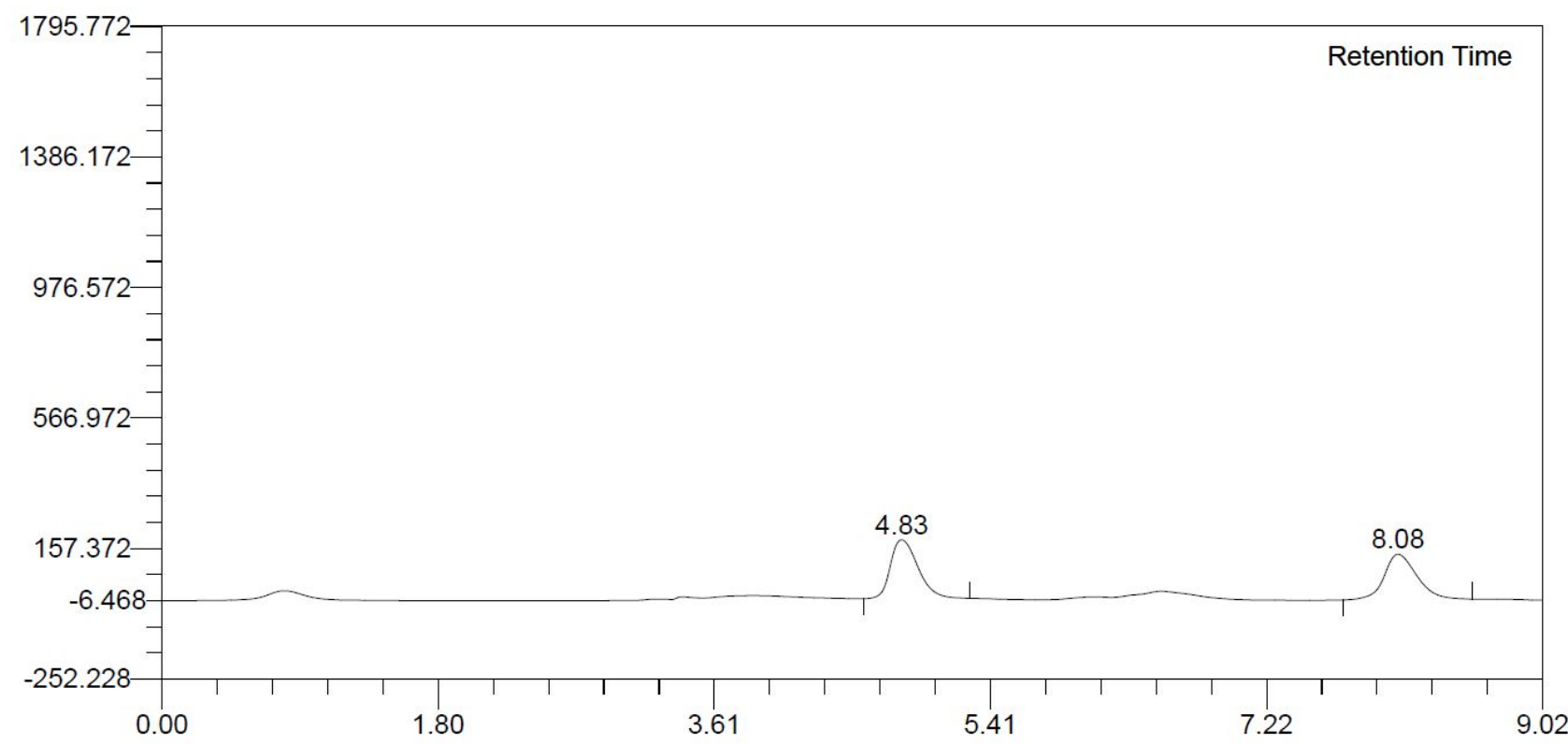

\begin{tabular}{|c|c|r|c|c|c|c|c|}
\hline PK No. & RT & Peak Width & Area & Height & Area $\%$ & Height $\%$ & Code \\
\hline 1 & 4.832 & 0.193 & 46060731 & 184290 & 52.19119 & 56.42820 & BB \\
2 & 8.076 & 0.220 & 42193118 & 142302 & 47.80881 & 43.57180 & BB \\
\hline
\end{tabular}

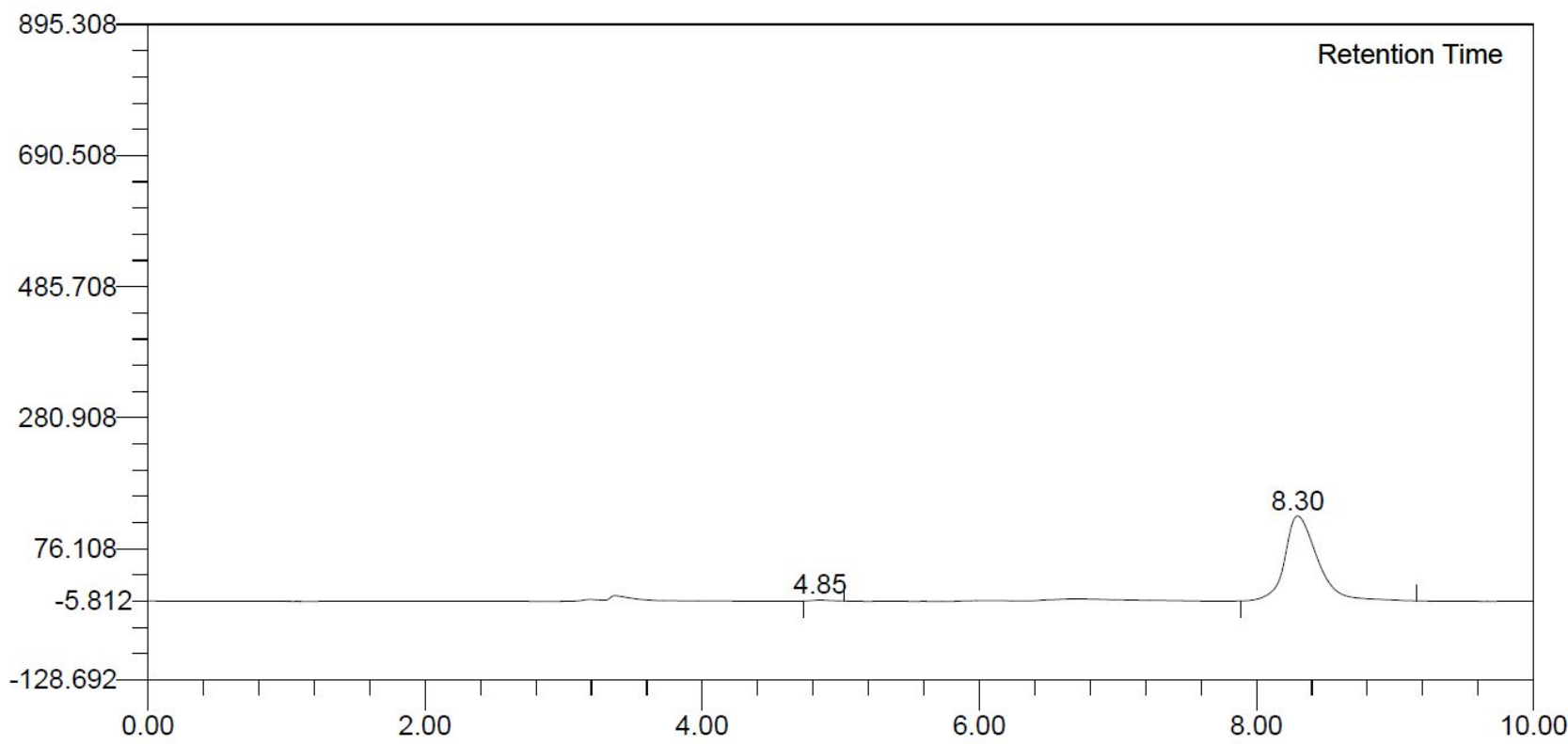

\begin{tabular}{|c|r|r|r|r|r|r|r|}
\hline PK No. & RT & Peak Width & \multicolumn{1}{c|}{ Area } & Height & Area \% & Height \% & Code \\
\hline 1 & 4.851 & 0.157 & 266401 & 1421 & 0.60616 & 1.05838 & BB \\
2 & 8.298 & 0.236 & 43682781 & 132841 & 99.39384 & 98.94162 & BB \\
\hline
\end{tabular}




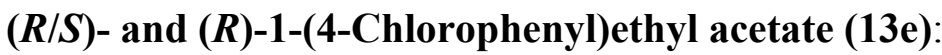

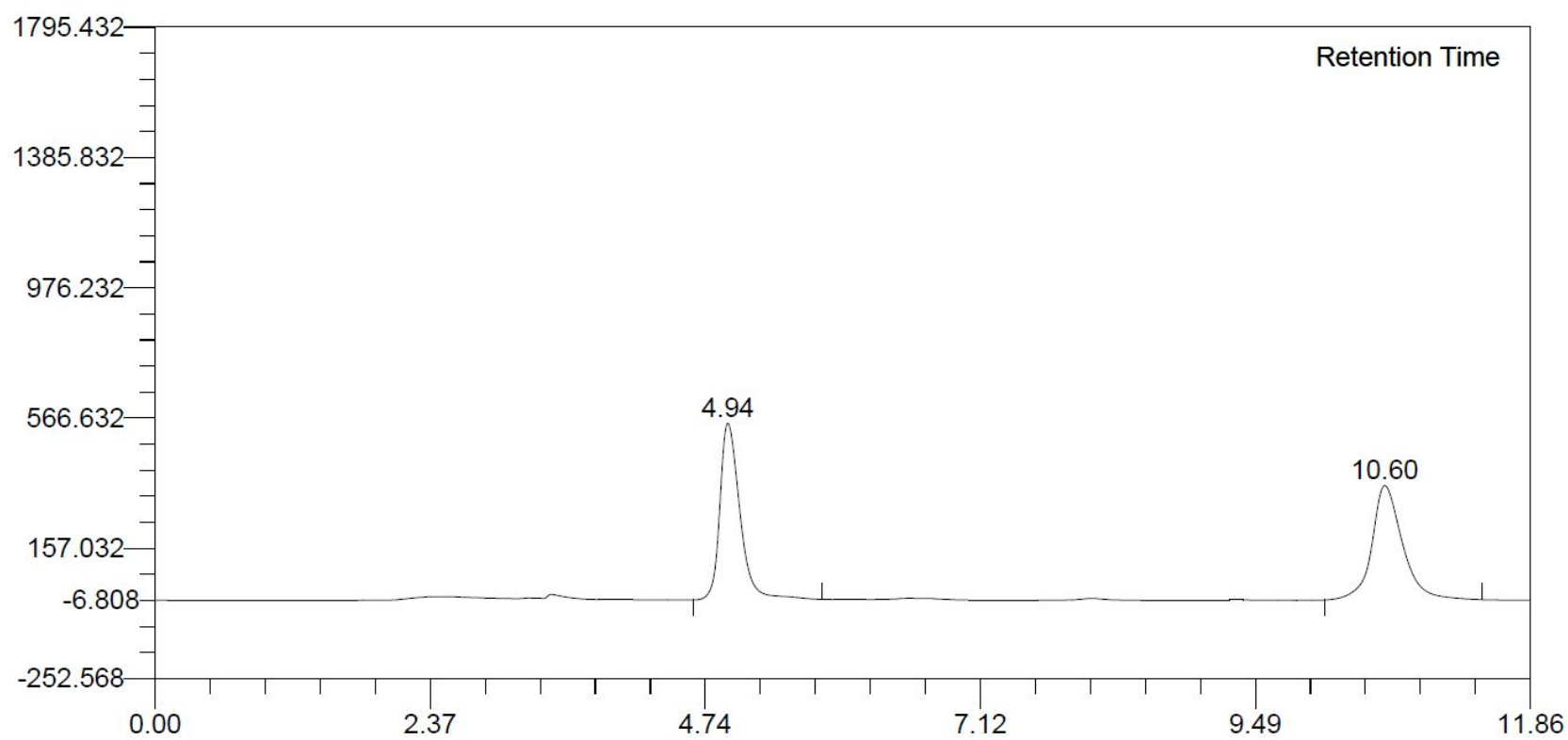

\begin{tabular}{|c|r|r|c|c|c|c|c|}
\hline PK No. & \multicolumn{1}{|c|}{ RT } & Peak Width & Area & Height & Area $\%$ & Height \% & Code \\
\hline 1 & 4.939 & 0.176 & 133949563 & 555010 & 50.38855 & 60.68601 & BB \\
2 & 10.604 & 0.261 & 131883787 & 359550 & 49.61145 & 39.31399 & BB \\
\hline
\end{tabular}

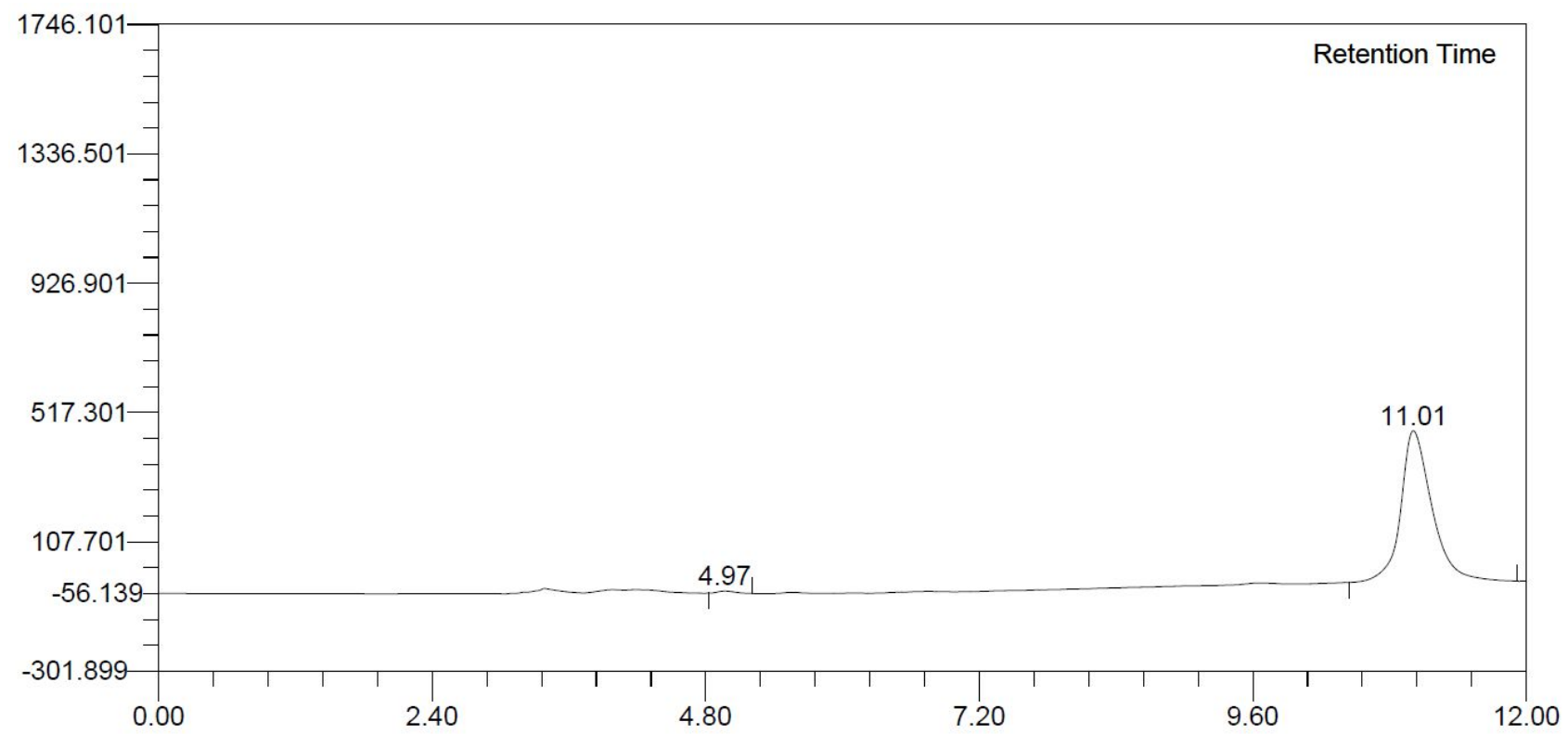

\begin{tabular}{|c|r|r|r|r|r|r|r|}
\hline PK No. & \multicolumn{1}{|c|}{ RT } & Peak Width & \multicolumn{1}{c|}{ Area } & Height & Area \% & Height $\%$ & Code \\
\hline 1 & 4.966 & 0.155 & 1443611 & 7017 & 0.78288 & 1.44442 & BB \\
2 & 11.010 & 0.269 & 182953117 & 478785 & 99.21712 & 98.55558 & BB \\
\hline
\end{tabular}


$(R / S)$ - and (R)-1-(4-Bromophenyl)ethyl acetate (13f):

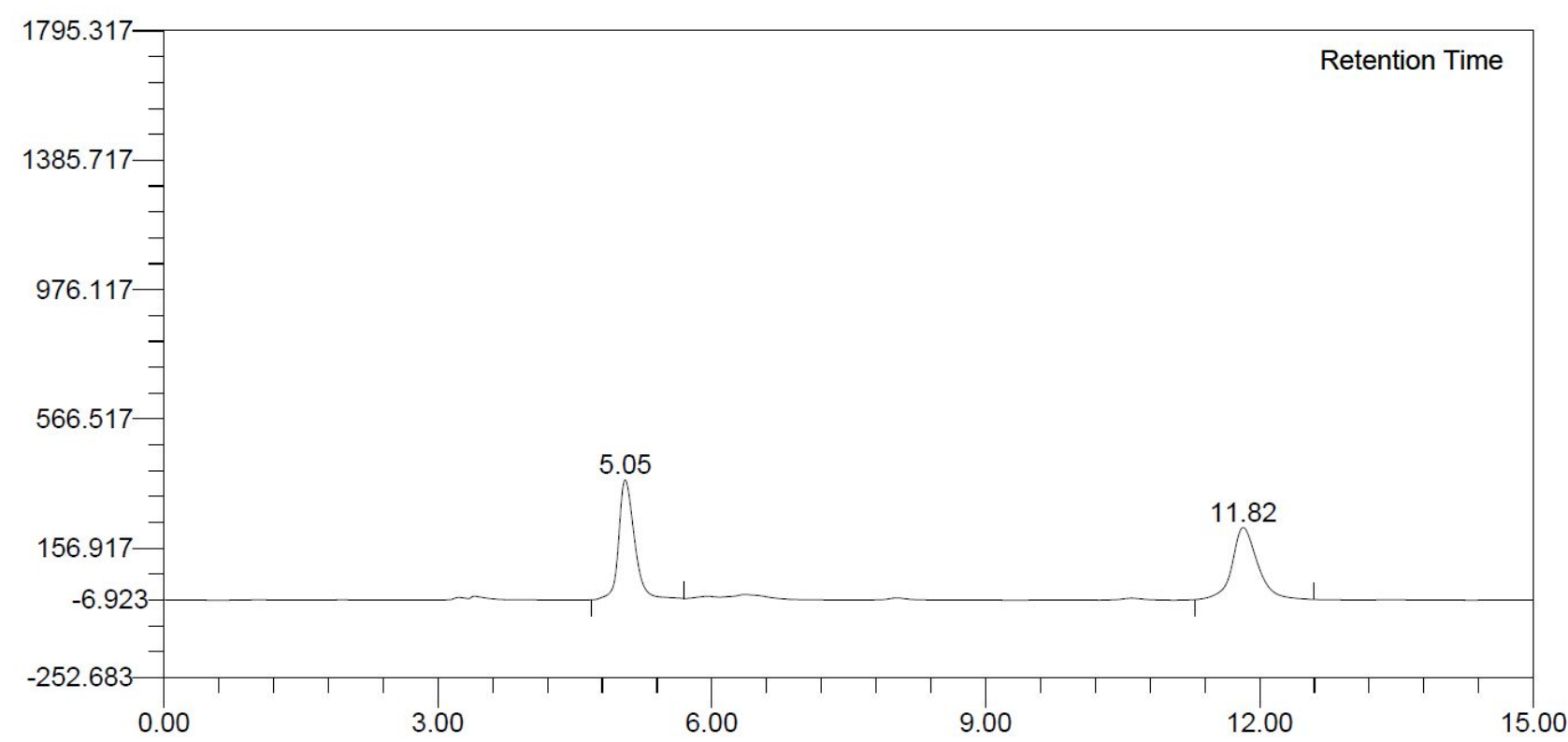

\begin{tabular}{|c|r|r|c|c|c|c|c|}
\hline PK No. & \multicolumn{1}{|c|}{ RT } & Peak Width & Area & Height & Area \% & Height $\%$ & Code \\
\hline 1 & 5.053 & 0.175 & 90655457 & 378153 & 50.51412 & 62.33421 & BB \\
2 & 11.822 & 0.278 & 88810112 & 228501 & 49.48588 & 37.66579 & BB \\
\hline
\end{tabular}

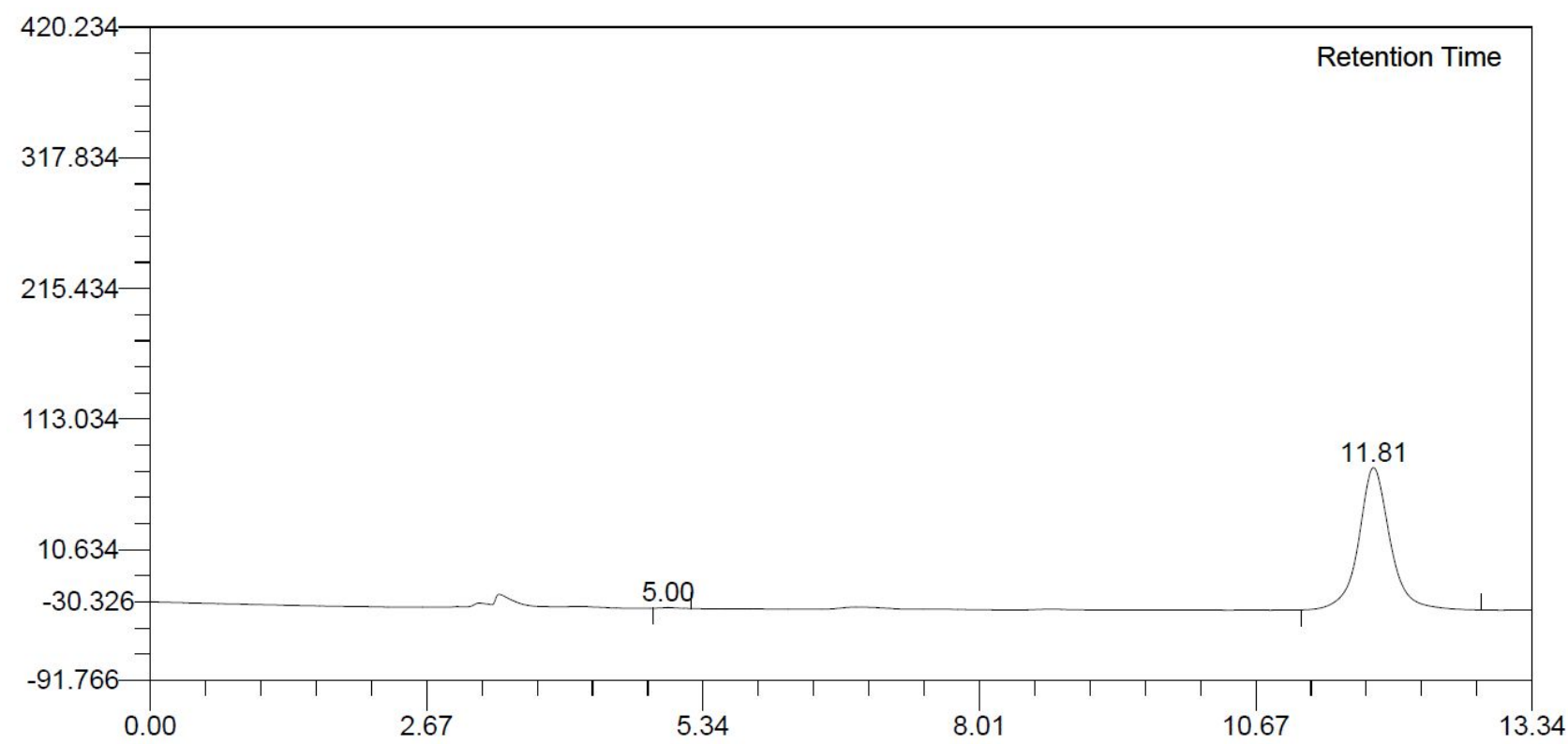

\begin{tabular}{|c|r|r|r|r|r|r|r|}
\hline PK No. & \multicolumn{1}{|c|}{ RT } & Peak Width & Area & Height & Area $\%$ & Height $\%$ & Code \\
\hline 1 & 5.001 & 0.145 & 123966 & 604 & 0.26156 & 0.53818 & BB \\
2 & 11.813 & 0.300 & 47270402 & 111626 & 99.73844 & 99.46182 & BB
\end{tabular}




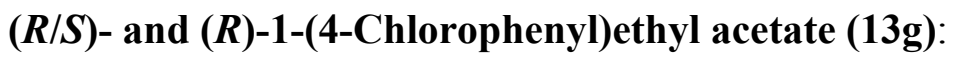

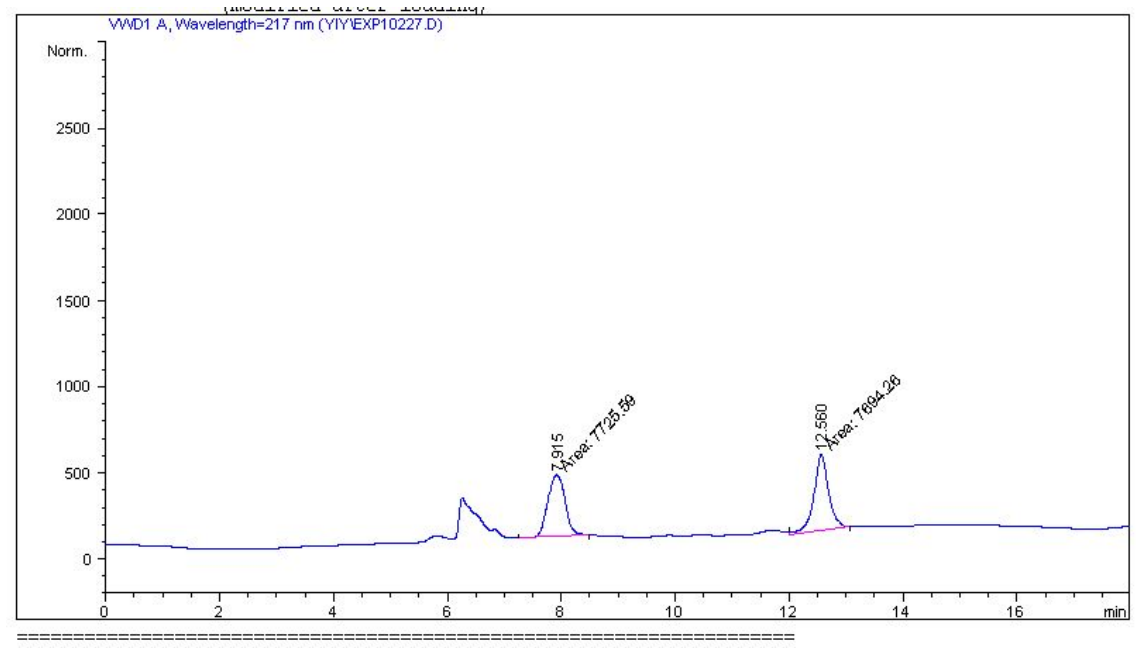

Area Percent Report

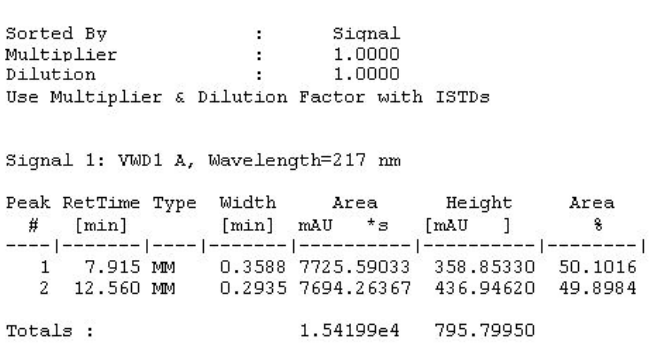

Results obtained with enhanced inteqrator!

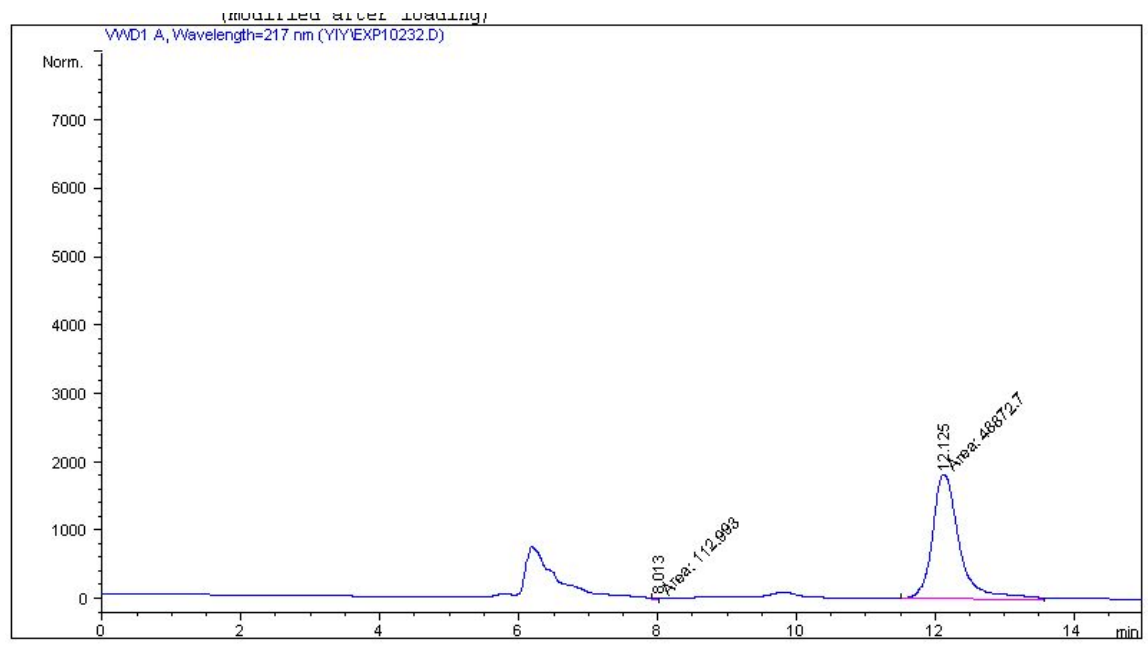

Area Percent Report

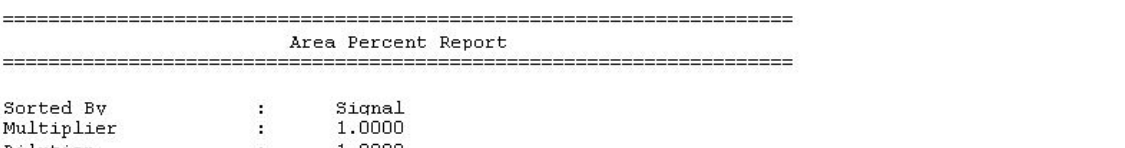

Multiplier

Use Multiplier \& Dilution Factor with ISTDs

Signal 1: VWD1 $\mathrm{A}$, Wavelength=217 $\mathrm{nm}$

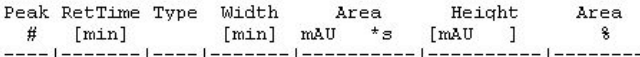

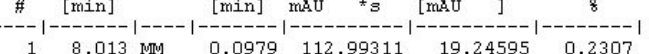

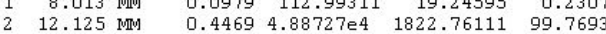

$$
\begin{aligned}
& \text { Totals : } \quad 4.89856 \mathrm{e} 4 \quad 1842.00706
\end{aligned}
$$

Results obtained with enhanced integrator!

$* * *$ End of Report $* * *$
$* *$ 
$(R / S)$ - and (R)-3-Chloro-1-phenylpropyl acetate (13h):

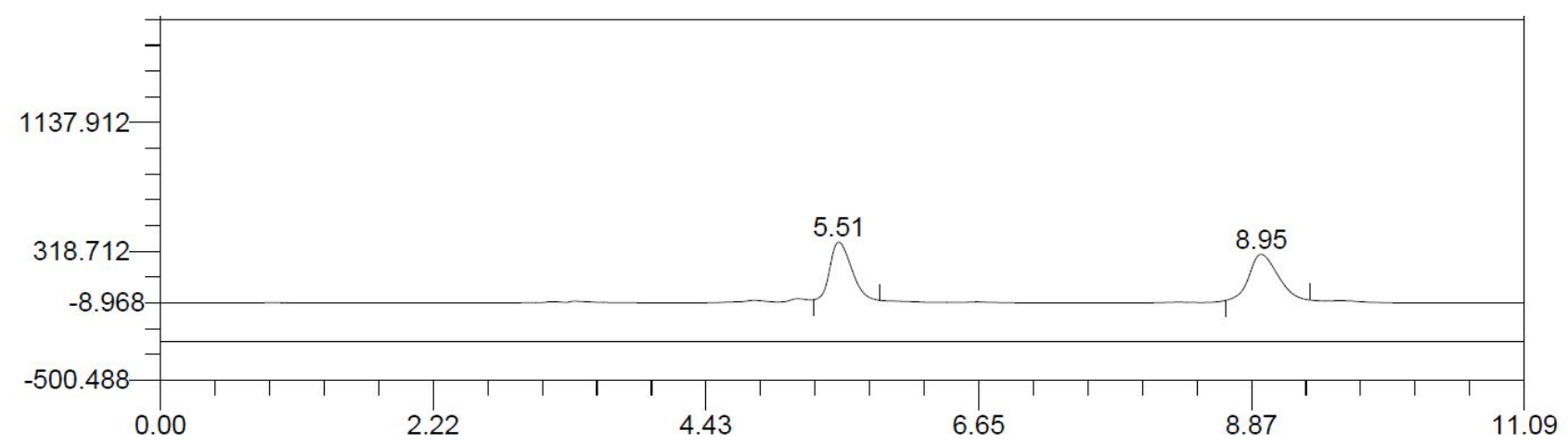

\begin{tabular}{|c|r|r|r|r|r|r|r|}
\hline PK No. & RT & Peak Width & Area & Height & Area \% & Height \% & Code \\
\hline 1 & 5.514 & 0.188 & 88678449 & 367844 & 48.47617 & 55.71672 & BB \\
2 & 8.948 & 0.248 & 94253581 & 292360 & 51.52383 & 44.28328
\end{tabular}

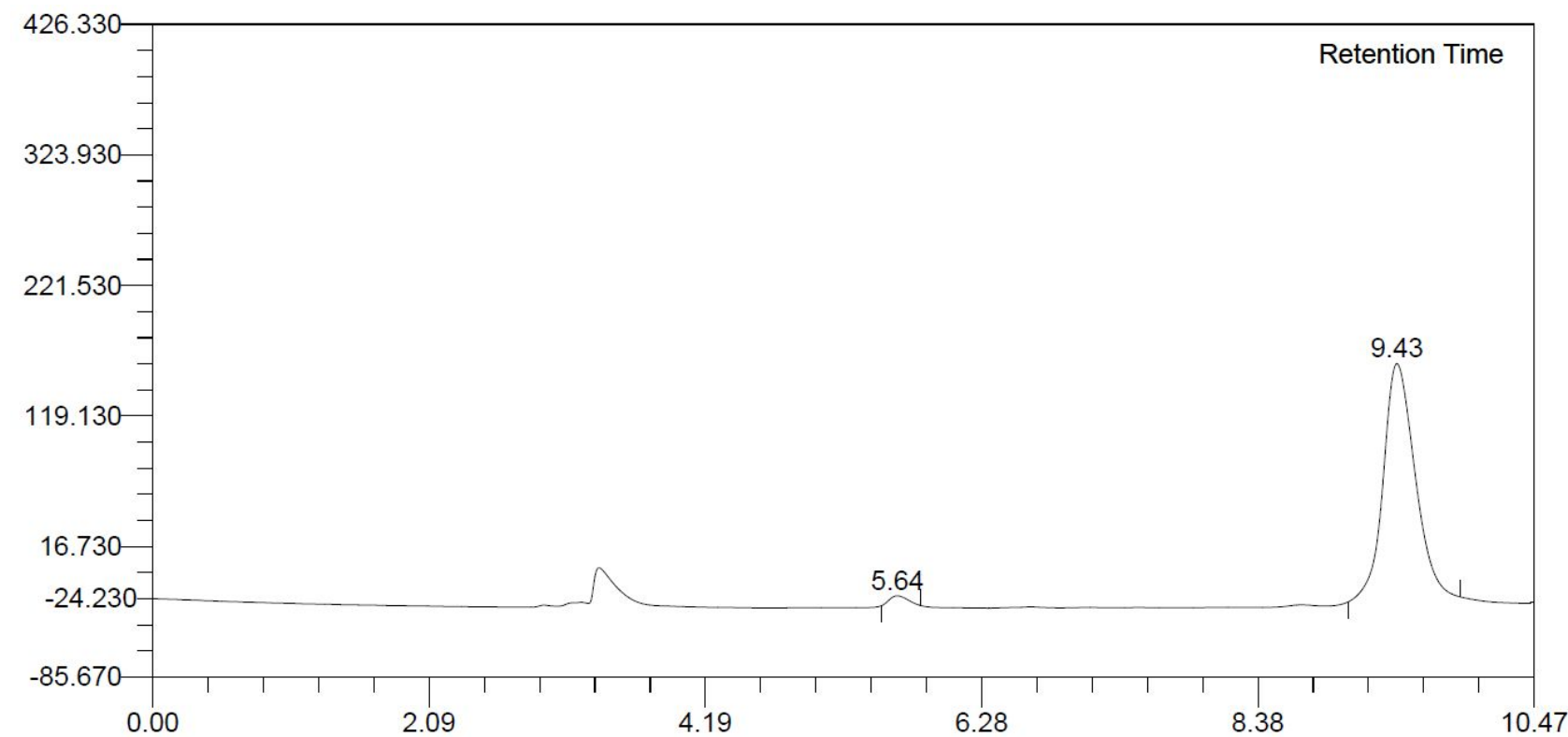

\begin{tabular}{|c|r|r|r|r|r|r|r|}
\hline PK No. & RT & Peak Width & \multicolumn{1}{c|}{ Area } & Height & \multicolumn{1}{|c|}{ Area \% } & Height \% & Code \\
\hline 1 & 5.643 & 0.162 & 1486650 & 7704 & 2.35141 & 3.99521 & BB \\
2 & 9.429 & 0.249 & 61736997 & 185127 & 97.64859 & 96.00479 & BB
\end{tabular}


$(R / S)$ - and $(R)-1-P h e n y l p r o p a n-2-y l$ acetate (13i):

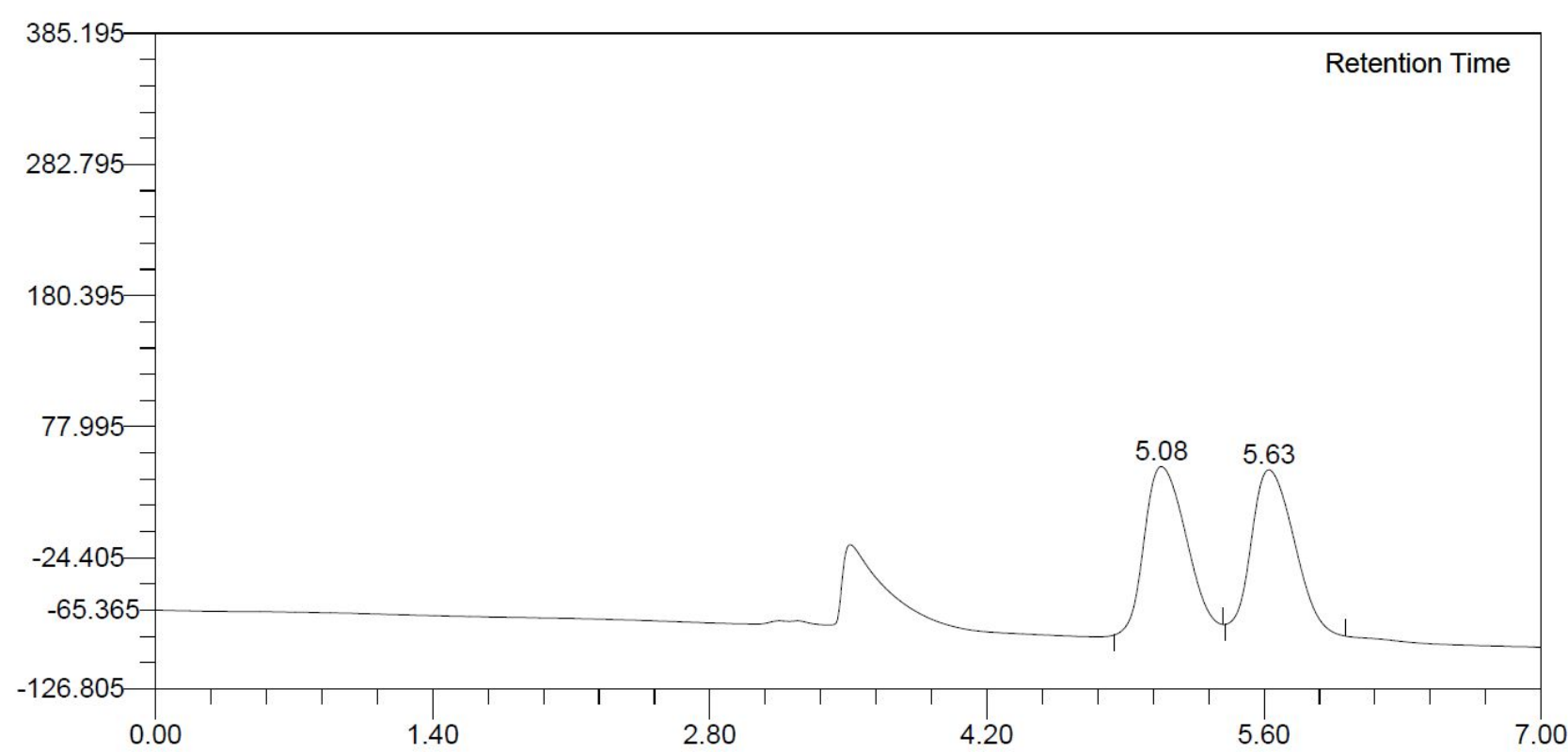

\begin{tabular}{|c|c|c|c|c|c|c|c|}
\hline PK No. & RT & Peak Width & Area & Height & Area \% & Height \% & Code \\
\hline 1 & 5.082 & 0.228 & 36370561 & 128004 & 50.01662 & 50.81197 & BB \\
2 & 5.625 & 0.230 & 36346391 & 123913 & 49.98338 & 49.18803 & BB \\
\hline
\end{tabular}

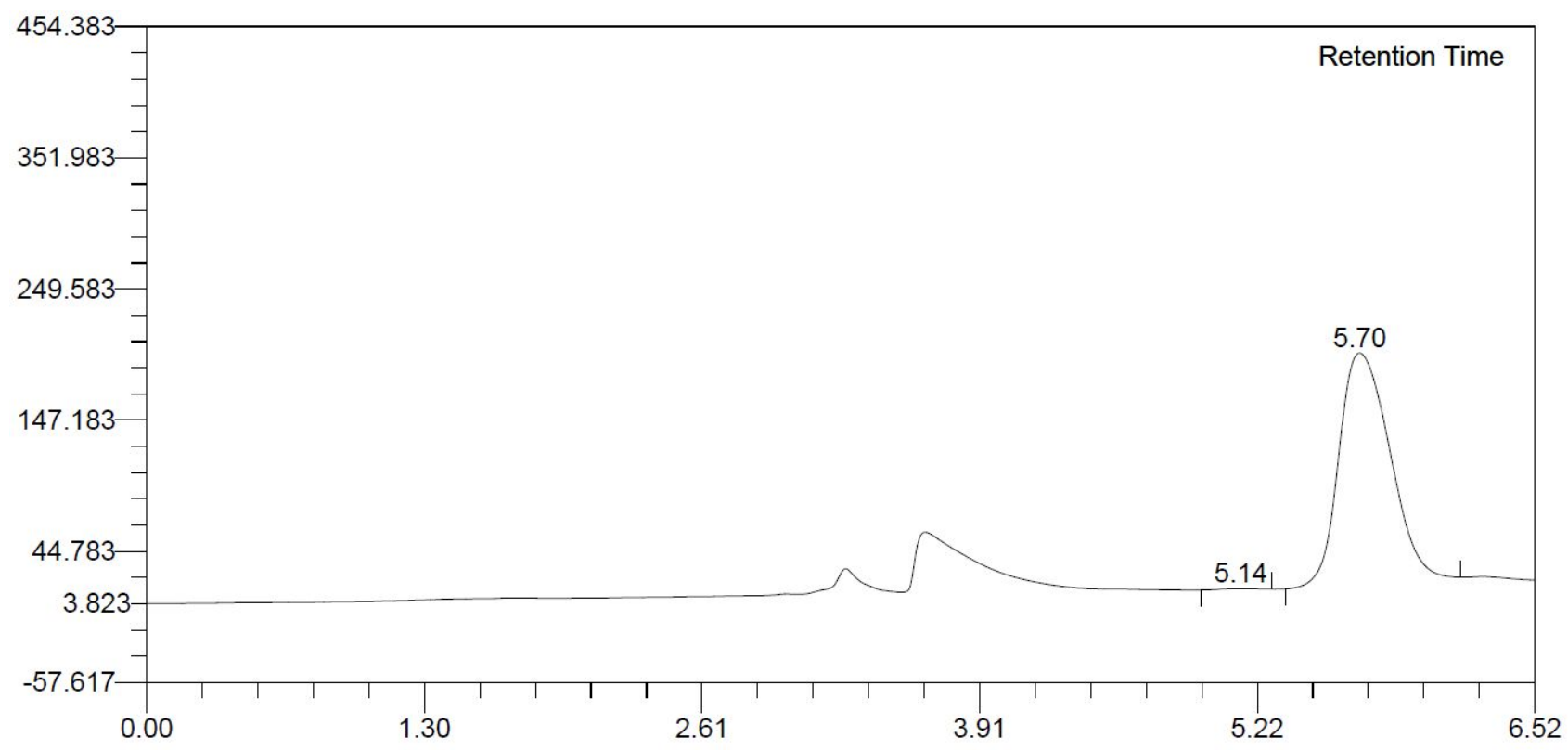

\begin{tabular}{|c|c|r|r|r|r|r|c|}
\hline PK No. & RT & Peak Width & Area & \multicolumn{1}{c|}{ Height } & Area $\%$ & Height $\%$ & Code \\
\hline 1 & 5.139 & 0.150 & 178796 & 799 & 0.29888 & 0.44119 & BB \\
2 & 5.698 & 0.260 & 59643440 & 180301 & 99.70112 & 99.55881 & BB \\
\hline
\end{tabular}


$(R / S)$ - and $(R)-4-P h e n y l b u t a n-2-y l$ acetate (13j):

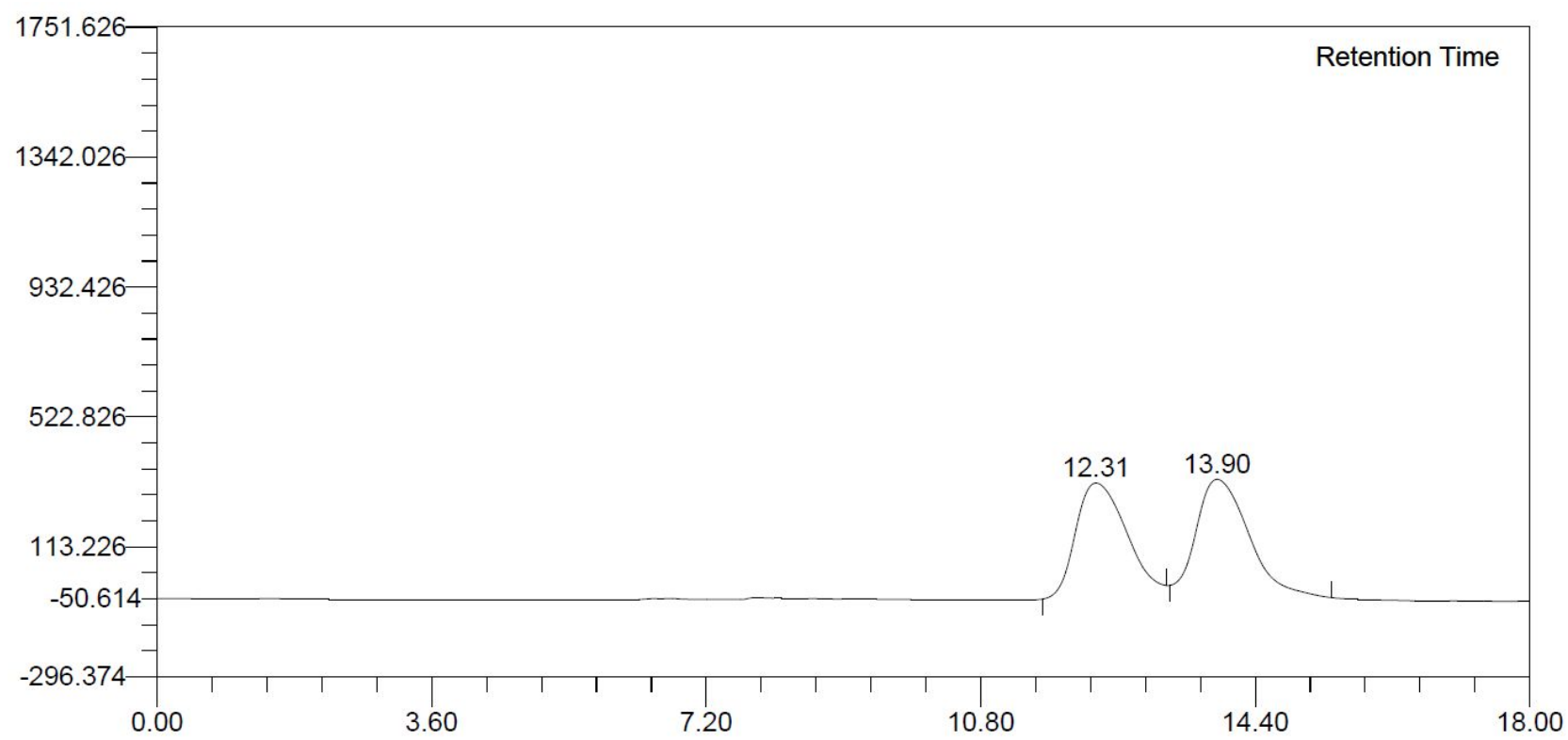

\begin{tabular}{|c|c|r|c|c|c|c|c|}
\hline PK No. & RT & Peak Width & Area & Height & Area \% & Height \% & Code \\
\hline 1 & 12.313 & 0.692 & 302126899 & 347158 & 49.16703 & 50.12251 & BB \\
2 & 13.901 & 0.689 & 312363986 & 345461 & 50.83297 & 49.87749 & BB \\
\hline
\end{tabular}

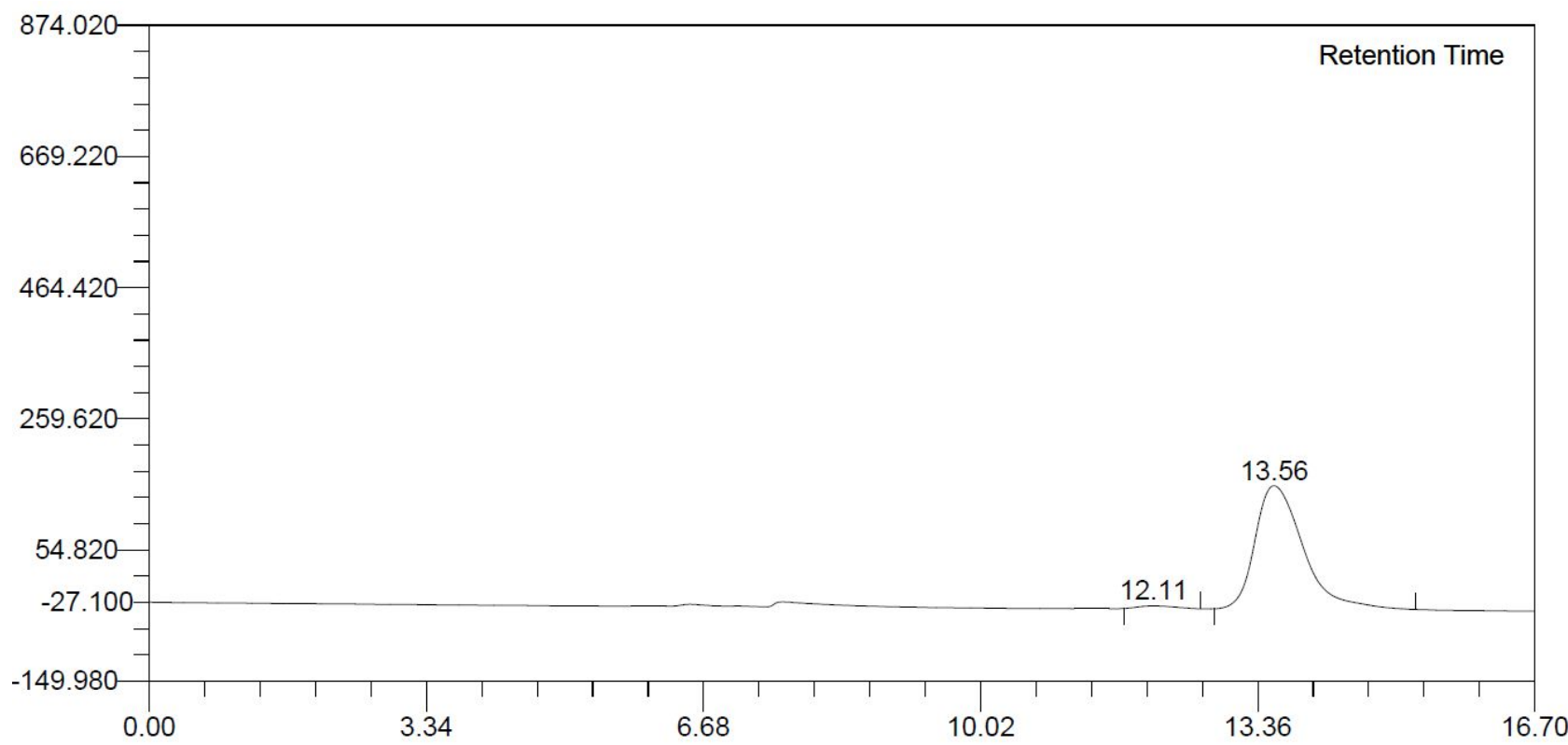

\begin{tabular}{|c|c|r|r|r|r|r|c|}
\hline PK No. & RT & Peak Width & Area & Height & Area $\%$ & Height $\%$ & Code \\
\hline 1 & 12.106 & 0.481 & 2540693 & 4225 & 1.61489 & 2.14689 & BB \\
2 & 13.557 & 0.599 & 154788658 & 192571 & 98.38511 & 97.85311 & BB \\
\hline
\end{tabular}


$(R / S)$ - and $(R)-1-($ Naphthalen-2-yl)ethyl acetate (13k):

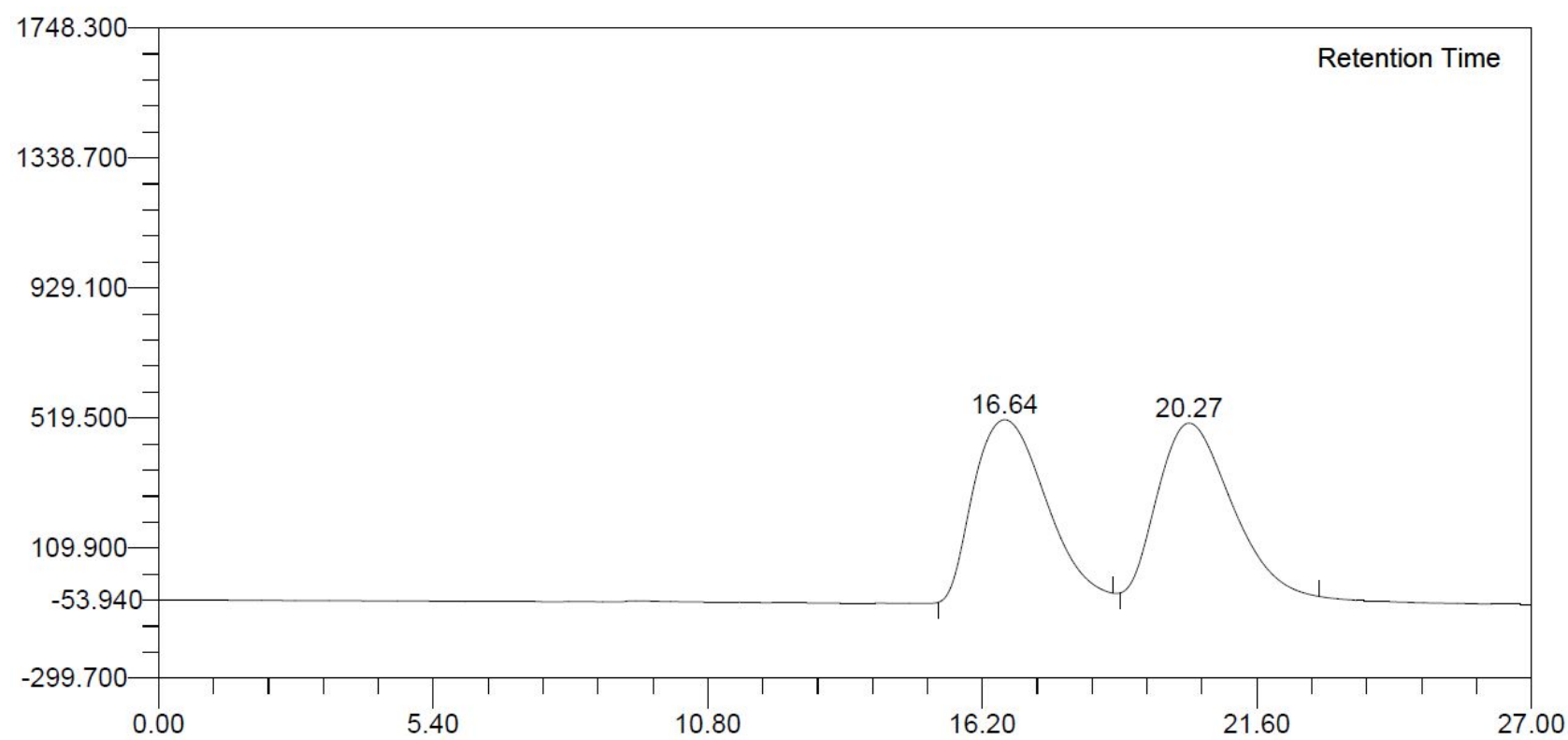

\begin{tabular}{|c|c|c|c|c|c|c|c|}
\hline PK No. & RT & Peak Width & Area & Height & Area \% & Height \% & Code \\
\hline 1 & 16.642 & 1.550 & 1086947388 & 563962 & 50.43230 & 51.15218 & BB \\
2 & 20.273 & 1.572 & 1068313036 & 538556 & 49.56770 & 48.84782 & $B B$
\end{tabular}

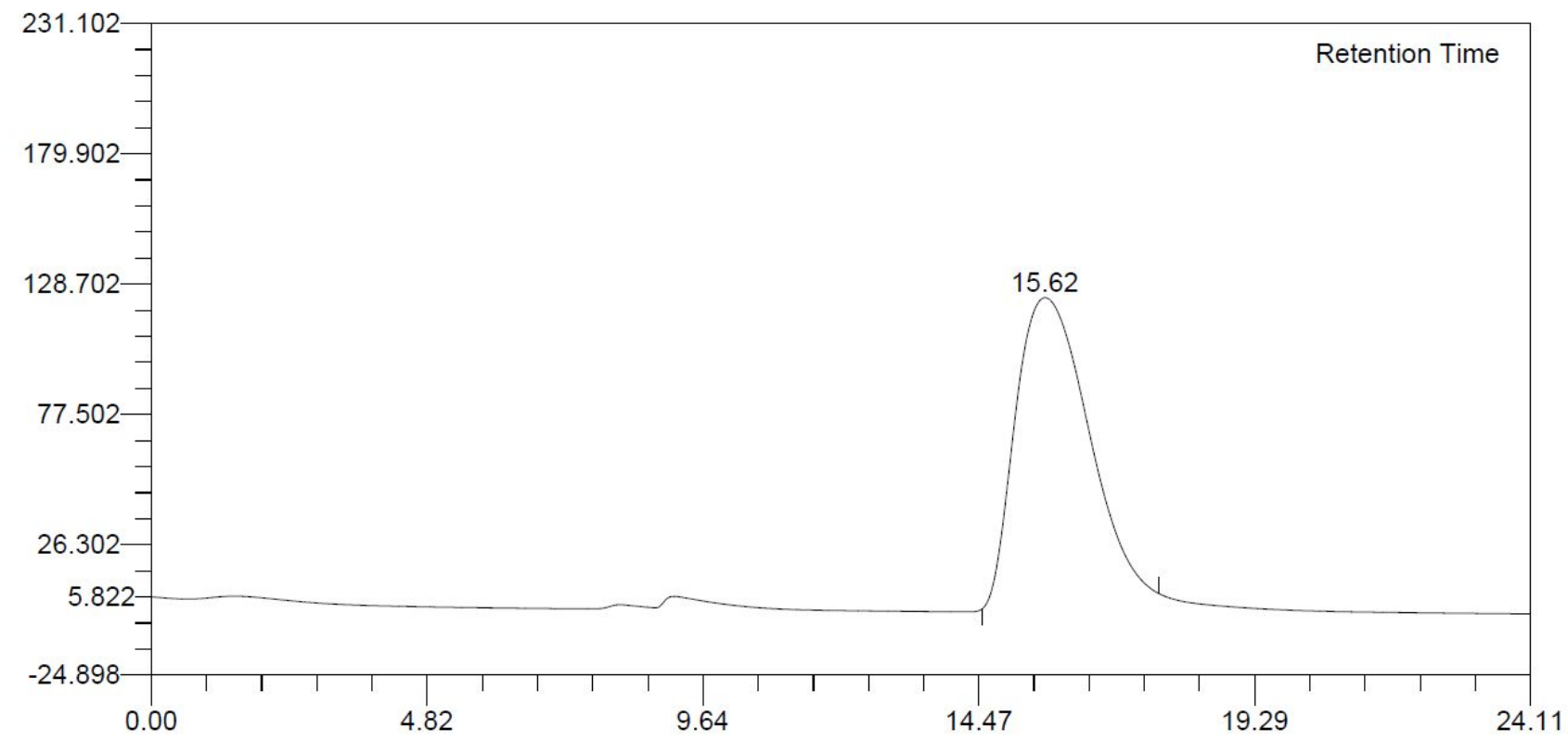

\begin{tabular}{|c|c|c|c|c|c|c|c|}
\hline PK No. & RT & Peak Width & Area & Height & Area $\%$ & Height $\%$ & Code \\
\hline 1 & 15.623 & 1.404 & 210461477 & 120081 & 100.00000 & 100.00000 & BB
\end{tabular}


$(R / S)$ - and (R)-1-Phenylallyl acetate (13I):

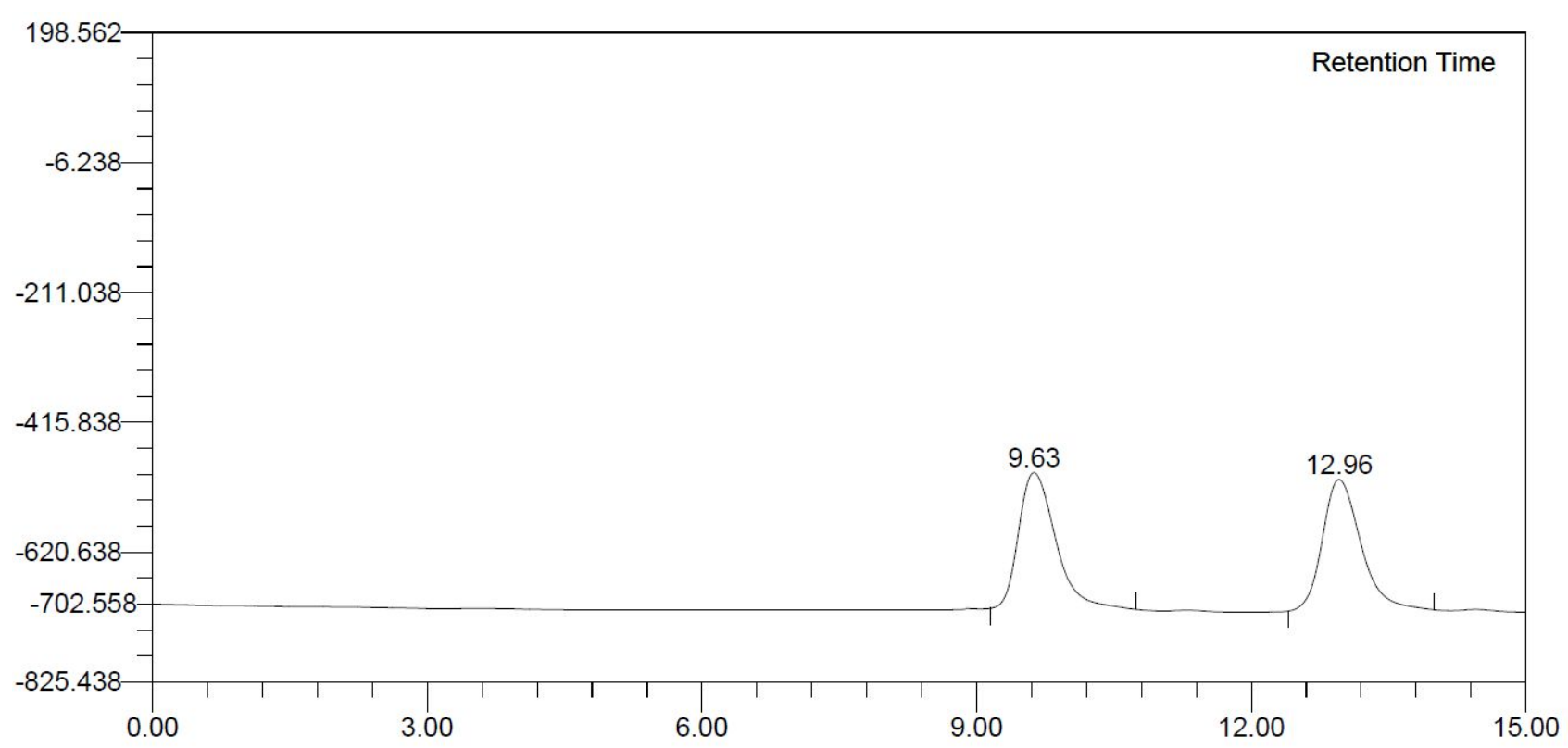

\begin{tabular}{|c|r|r|c|c|c|c|c|}
\hline PK No. & \multicolumn{1}{|c|}{ RT } & Peak Width & Area & Height & Area $\%$ & Height $\%$ & Code \\
\hline 1 & 9.627 & 0.424 & 120788491 & 214457 & 50.07207 & 50.92309 & BB \\
2 & 12.959 & 0.436 & 120440788 & 206682 & 49.92793 & 49.07691 & BB
\end{tabular}

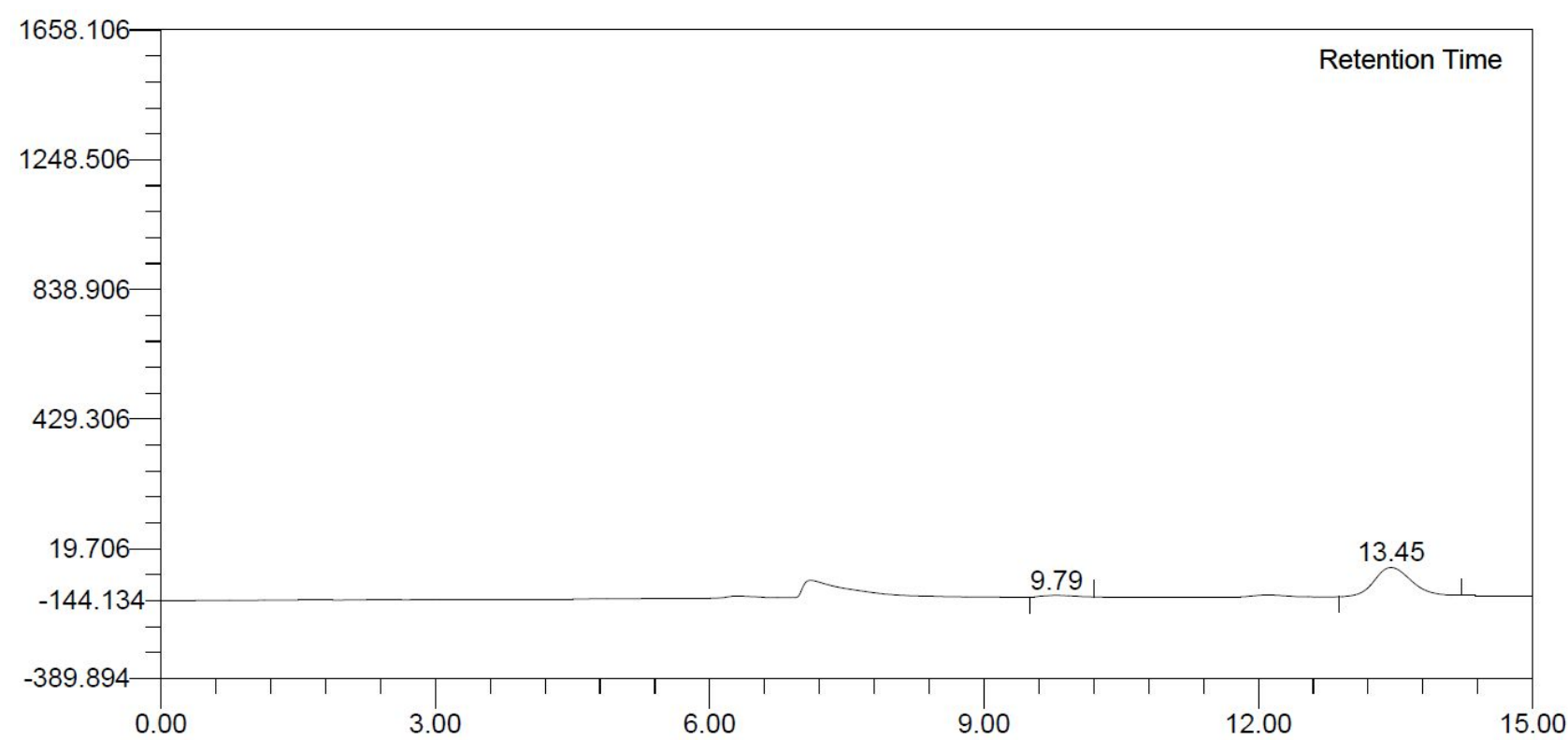

\begin{tabular}{|c|r|r|r|r|r|r|r|}
\hline PK No. & \multicolumn{1}{|c|}{ RT } & Peak Width & \multicolumn{1}{c|}{ Area } & Height & \multicolumn{1}{|c|}{ Area \% } & Height \% & Code \\
\hline 1 & 9.793 & 0.367 & 2081525 & 4702 & 3.94233 & 4.92320 & BB \\
2 & 13.452 & 0.422 & 50717855 & 90805 & 96.05767 & 95.07680 & BB
\end{tabular}


$(R / S)-$ and $(R)-1-(p$-Tolyl)allyl acetate (13m):

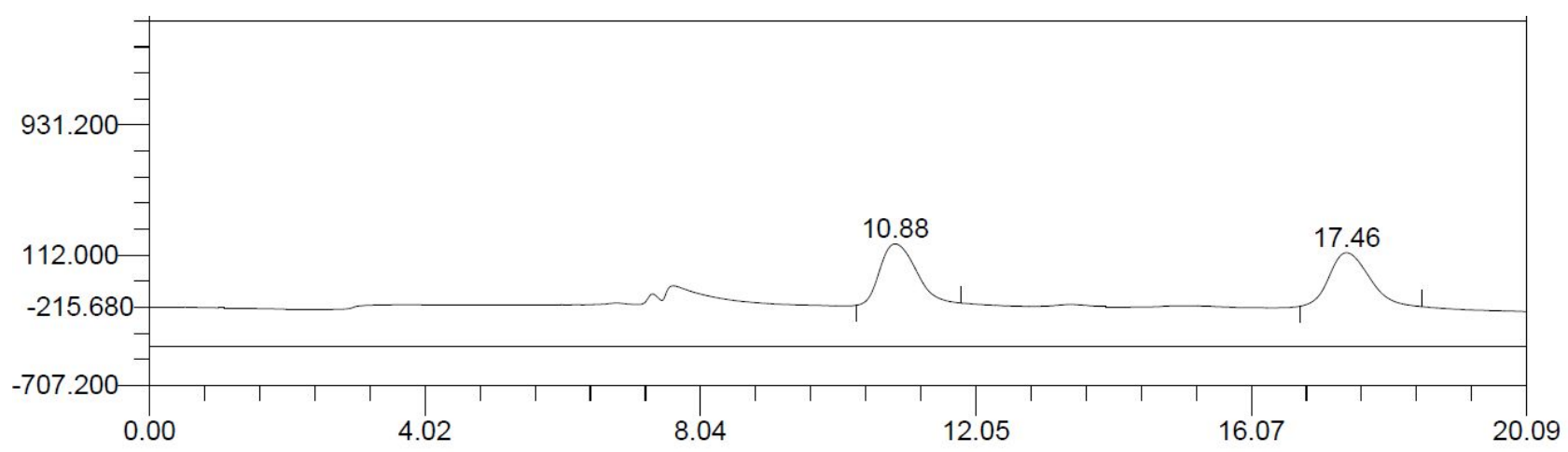

\begin{tabular}{|c|c|r|c|c|c|c|c|}
\hline PK No. & RT & Peak Width & Area & Height & Area $\%$ & Height \% & Code \\
\hline 1 & 10.877 & 0.571 & 276588050 & 380725 & 50.20688 & 52.96889 & BB \\
2 & 17.462 & 0.629 & 274308614 & 338046 & 49.79312 & 47.03111 & BB \\
\hline
\end{tabular}

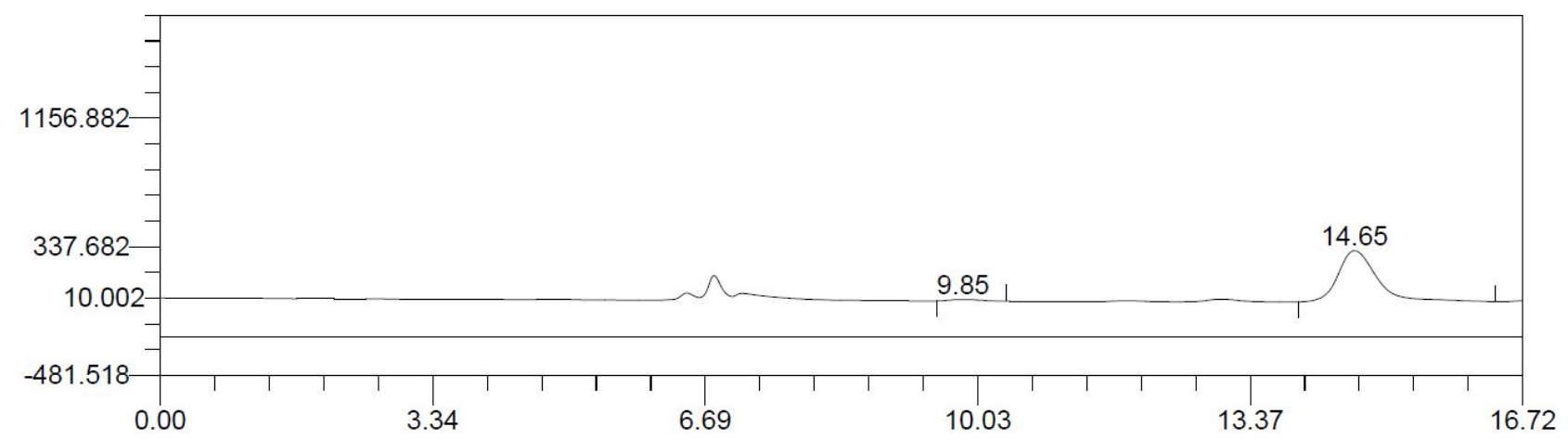

\begin{tabular}{|c|r|r|r|r|r|r|r|}
\hline PK No. & \multicolumn{1}{|c|}{ RT } & Peak Width & \multicolumn{1}{c|}{ Area } & Height & Area $\%$ & Height $\%$ & Code \\
\hline 1 & 9.848 & 0.401 & 4766270 & 9209 & 2.16278 & 2.75141 & BB \\
2 & 14.653 & 0.473 & 215611038 & 325492 & 97.83722 & 97.24859 & BB \\
\hline
\end{tabular}


$(R / S)$ - and (R)-1-(4-Methoxyphenyl)allyl acetate (13n):

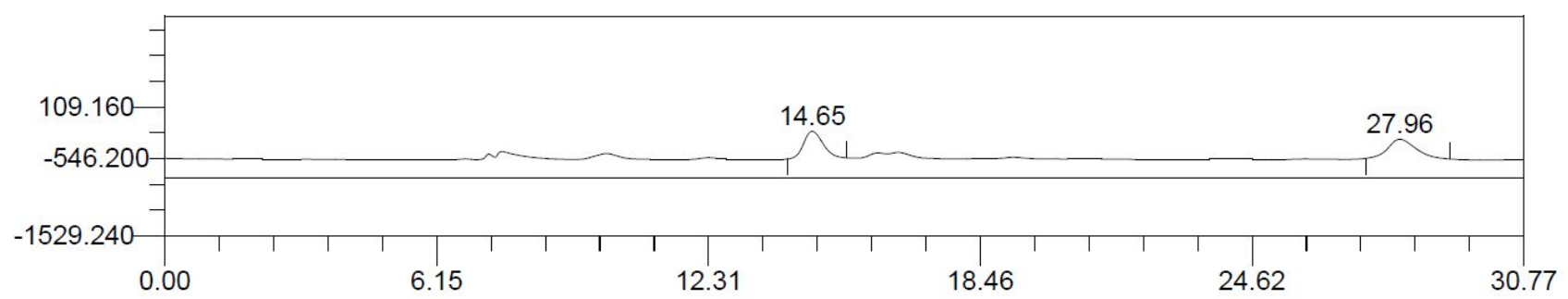

\begin{tabular}{|c|c|c|c|c|c|c|c|}
\hline PK No. & RT & Peak Width & Area & Height & Area \% & Height \% & Code \\
\hline 1 & 14.654 & 0.465 & 211942700 & 347633 & 49.49930 & 58.57377 & BB \\
2 & 27.962 & 0.654 & 216230412 & 245863 & 50.50070 & 41.42623 & BB
\end{tabular}

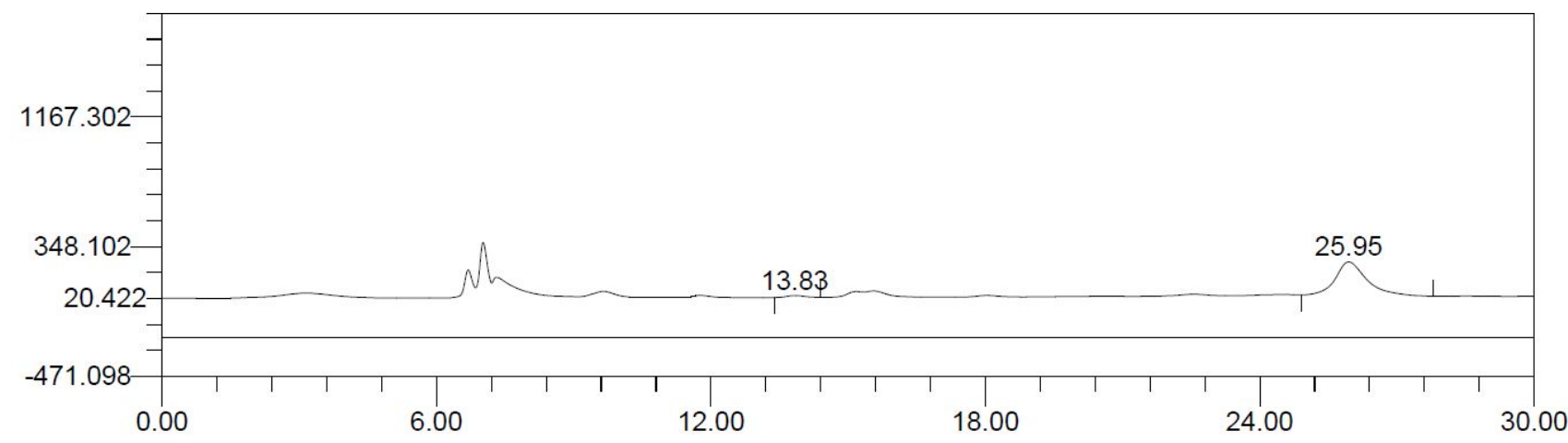

\begin{tabular}{|c|c|r|r|r|r|r|r|}
\hline PK No. & RT & Peak Width & \multicolumn{1}{c|}{ Area } & Height & \multicolumn{1}{|c|}{ Area \% } & Height \% & Code \\
\hline 1 & 13.833 & 0.403 & 6596341 & 12526 & 3.15888 & 5.59441 & BB \\
2 & 25.946 & 0.660 & 202222350 & 211376 & 96.84112 & 94.40559 & BB
\end{tabular}


$(R / S)$ - and (R)-1-(4-Chlorophenyl)allyl acetate (130):

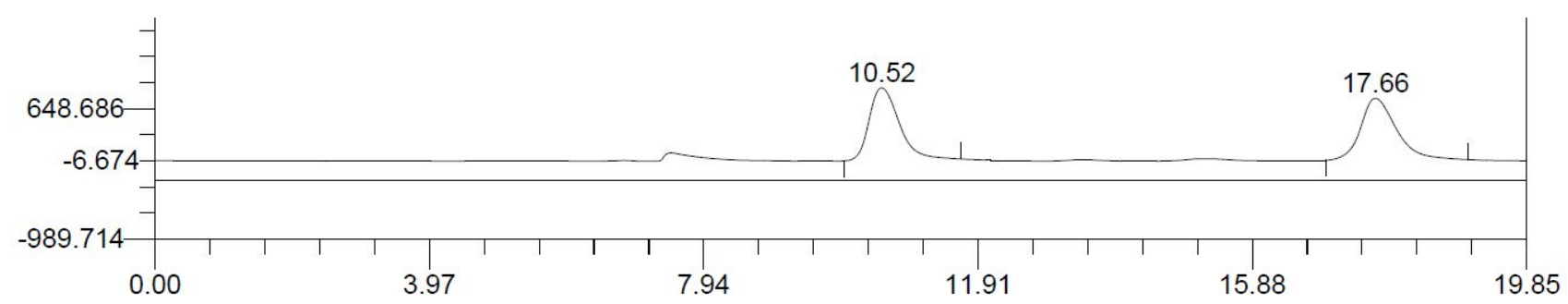

\begin{tabular}{|c|c|c|c|c|c|c|c|}
\hline PK No. & RT & Peak Width & Area & Height & Area \% & Height \% & Code \\
\hline 1 & 10.516 & 0.453 & 546929245 & 910077 & 49.51524 & 53.94203 & BB \\
2 & 17.662 & 0.524 & 557638308 & 777062 & 50.48476 & 46.05797 & BB
\end{tabular}

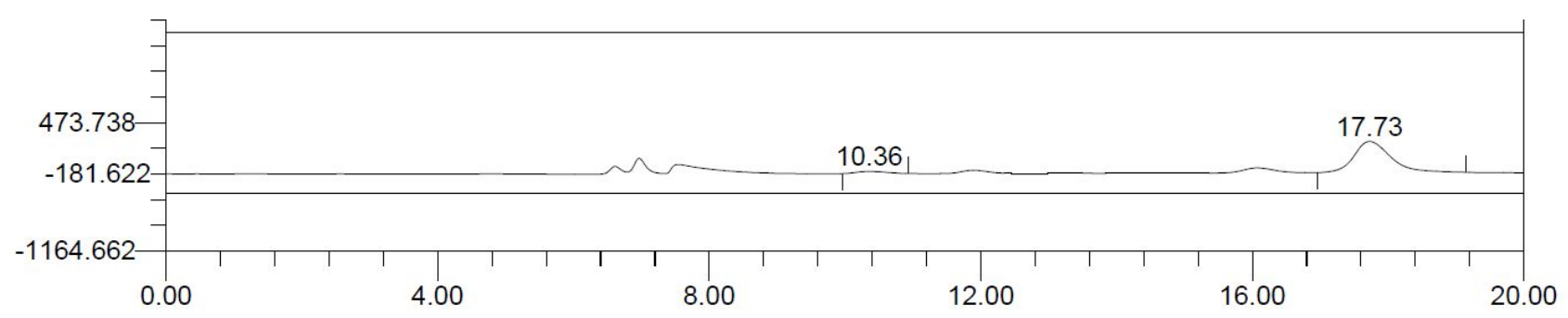

\begin{tabular}{|c|c|r|r|r|r|r|r|}
\hline PK No. & RT & Peak Width & Area & Height & Area \% & Height \% & Code \\
\hline 1 & 10.358 & 0.477 & 16067300 & 27432 & 5.11685 & 6.46437 & BB \\
2 & 17.727 & 0.549 & 297940220 & 396925 & 94.88315 & 93.53563 & BB
\end{tabular}


$(R / S)$ - and (R)-1-(4-Bromophenyl)allyl acetate (13p):

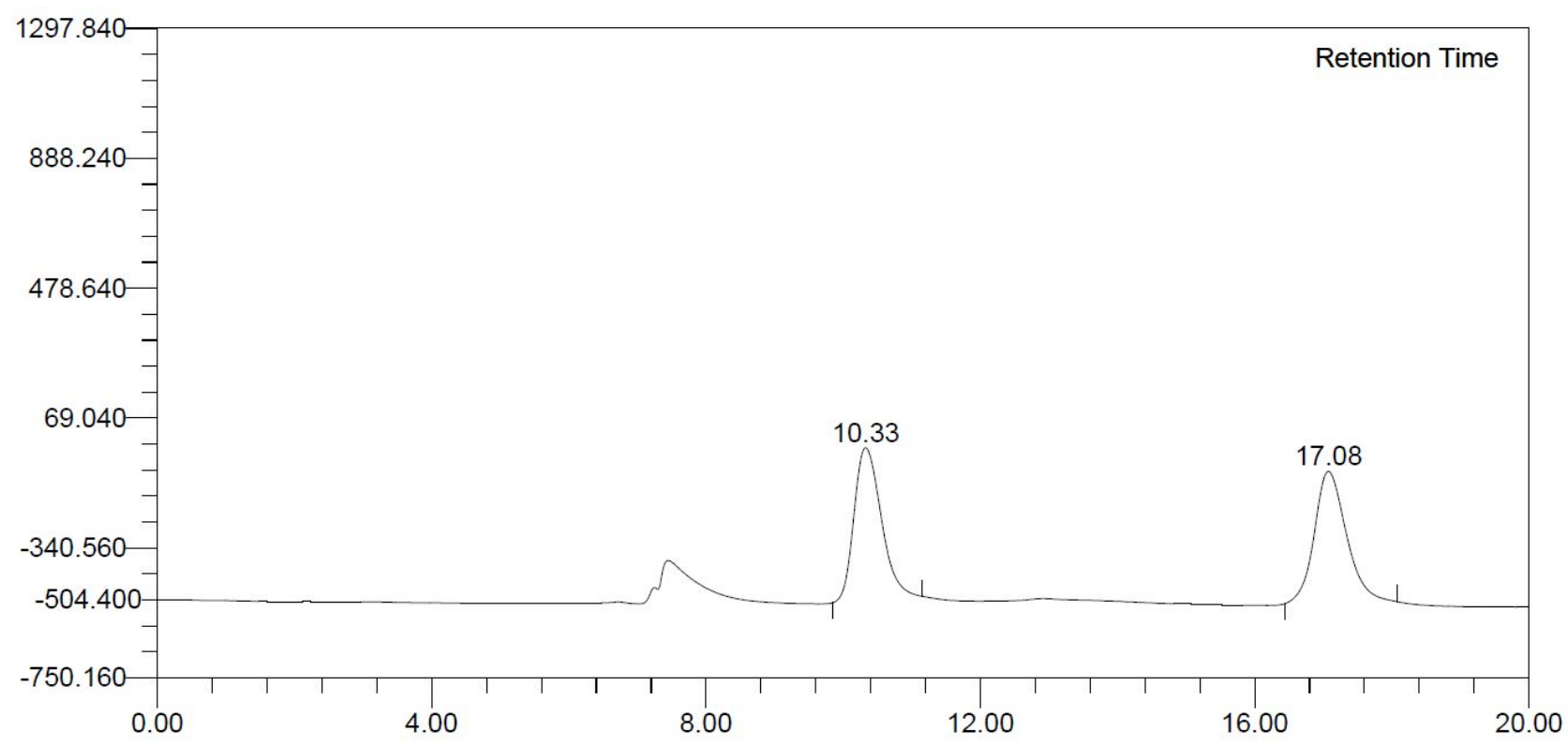

\begin{tabular}{|c|c|c|c|c|c|c|c|}
\hline PK No. & RT & Peak Width & Area & Height & Area \% & Height \% & Code \\
\hline 1 & 10.329 & 0.421 & 265439218 & 481401 & 49.57517 & 53.72018 & BB \\
2 & 17.077 & 0.485 & 269988580 & 414726 & 50.42483 & 46.27982 & $B B$
\end{tabular}

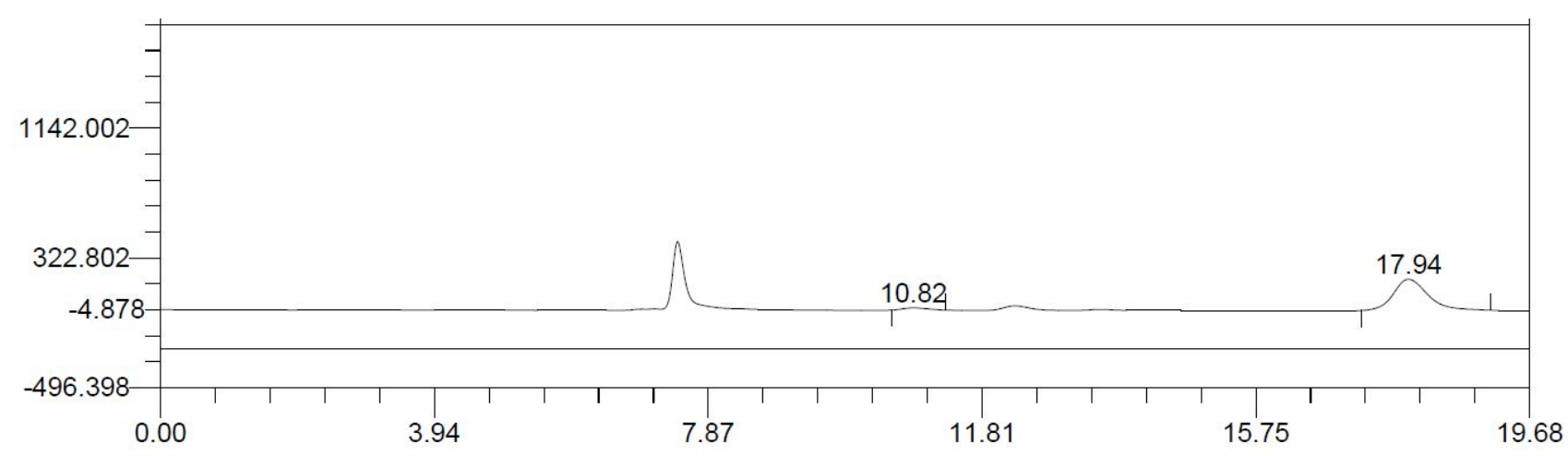

\begin{tabular}{|c|c|r|r|r|r|r|r|}
\hline PK No. & RT & Peak Width & Area & Height & \multicolumn{1}{|c|}{ Area \% } & Height \% & Code \\
\hline 1 & 10.823 & 0.403 & 6658894 & 13723 & 4.67390 & 6.51083 & BB \\
2 & 17.941 & 0.507 & 135810999 & 197049 & 95.32610 & 93.48917 & BB
\end{tabular}


$(R / S)$ - and $(R, E)-4-p h e n y l b u t-3-e n-2-y l$ acetate $(13 q)$ :

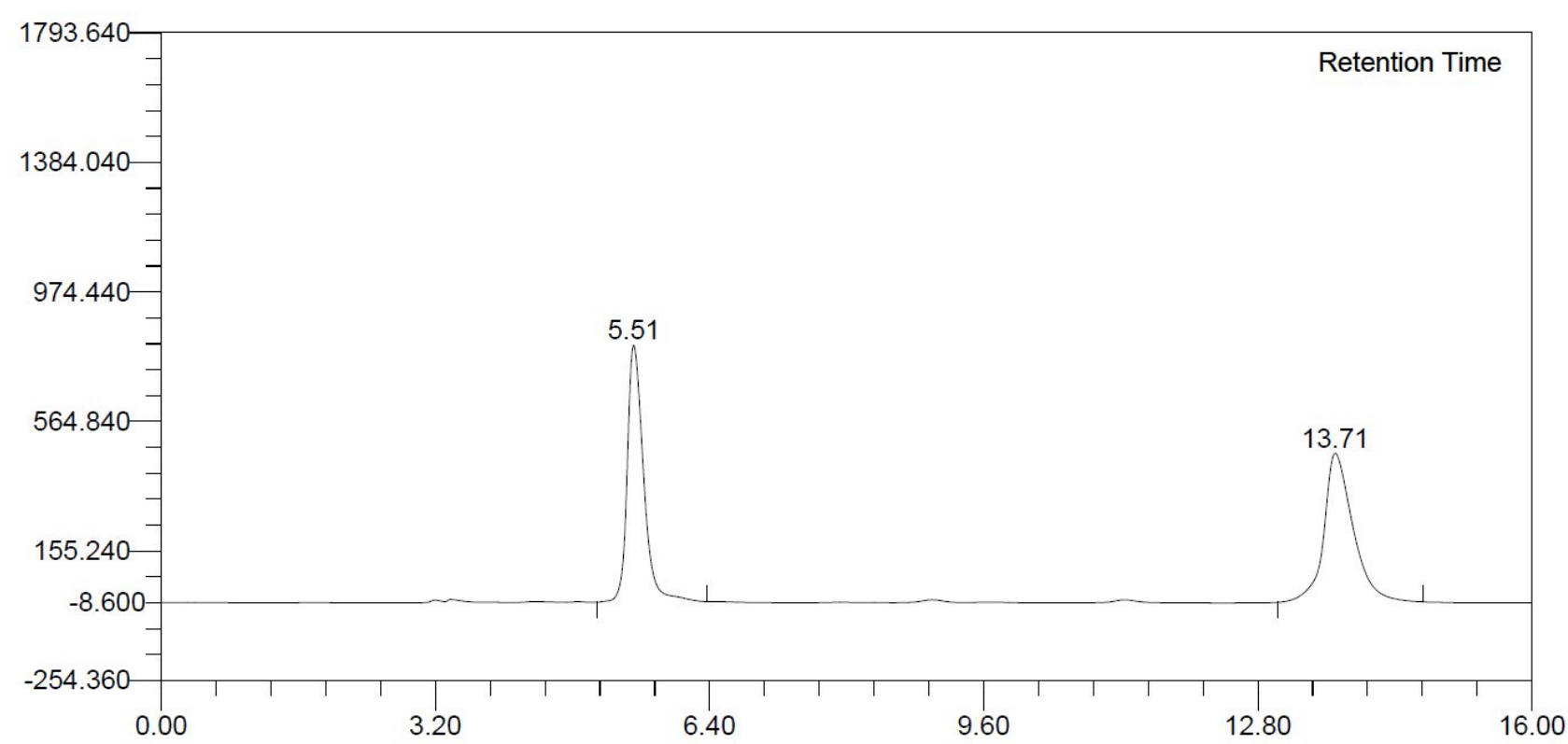

\begin{tabular}{|c|r|r|c|c|c|c|c|}
\hline PK No. & \multicolumn{1}{|c|}{ RT } & Peak Width & Area & Height & Area \% & Height \% & Code \\
\hline 1 & 5.515 & 0.193 & 215802894 & 811667 & 49.14832 & 63.29429 & BB \\
2 & 13.705 & 0.335 & 223282066 & 470703 & 50.85168 & 36.70571 & BB
\end{tabular}

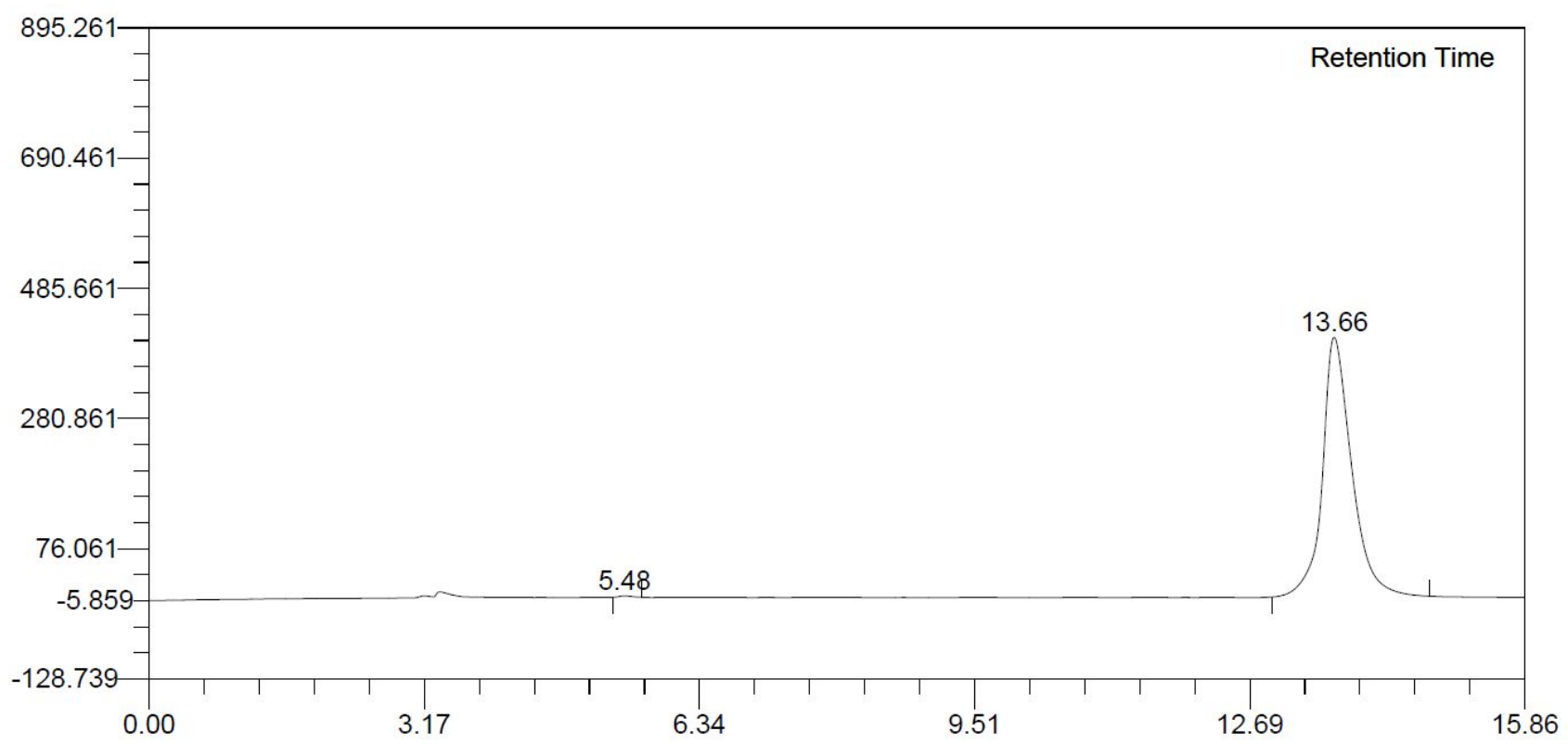

\begin{tabular}{|c|r|r|r|r|r|r|c|}
\hline PK No. & \multicolumn{1}{|c|}{ RT } & Peak Width & \multicolumn{1}{c|}{ Area } & Height & Area $\%$ & Height $\%$ & Code \\
\hline 1 & 5.480 & 0.169 & 465546 & 2279 & 0.24214 & 0.55530 & BB \\
2 & 13.660 & 0.331 & 191798203 & 408129 & 99.75786 & 99.44470 & BB \\
\hline
\end{tabular}

